Japan. J. Math.

Vol. 21, No. 2, 1995

\title{
Calcul stochastique d'ordre deux et équation différentielle anticipative sur une variété
}

\author{
By Axel Grorud and Monique Pontier \\ (Received April 6, 1993) \\ (Revised May 25, 1994)
}

\subsection{Introduction}

Ce travail a été motivé par l'étude de l'existence de solutions d'équations différentielles stochastiques anticipatives à valeurs dans une variété compacte $V$. Plus précisément, il s'agit d'équations différentielles stochastiques dont la condition initiale est une variable aléatoire "anticipante", c'est à dire mesurable par rapport à la tribu générale $\mathcal{F}$. Le problème des équations différentielles stochastiques "anticipantes" a déjà été étudié, du moins dans le cas vectoriel, par Buckdahn [1] d'une part, et Ocone et Pardoux d'autre part dans [18], où il s'agit d'équations linéaires, et dans [17] que nous généralisons ici au cas des variétés.

Selon la terminologie de Emery [4], on dit que le processus $X$ à valeurs dans la variété $V$ est solution de l'E.D.S.

$$
d X_{t}=e\left(B_{t}, X_{t}\right) \circ d B_{t}, \quad \text { avec } \quad X_{0} \in V
$$

où $B$ est le mouvement Brownien sur $V$ ([2] ou [9]), si pour toute 1-forme $\eta$ sur $V$, on a:

$$
\int_{0}^{t} \eta\left(X_{s}\right) \circ d X_{s}=\int_{0}^{t} e^{*}\left(B_{s}, X_{s}\right) \eta\left(B_{s}\right) \circ d B_{s} .
$$

Dans l'égalité ci-dessus, lorsque $X_{0} \in \mathcal{F}$, à droite on intègre une 1-forme anticipative le long de $B$ ce qui a été défini dans [8]; à gauche de l'égalité on doit intégrer une 1-forme anticipative le long d'un processus anticipatif, ce que nous étudions dans cet article. Les résultats obtenus sont à rapprocher du travail de Jolis et Sanz [10] puisque l'on obtient des conditions d'intégrabilité de processus anticipants par rapport à une semi-martingale intégrateur de type Skorohod aussi bien applicables à des processus vectoriels.

Nous suivons l'approche de Emery [4] qui intègre des formes différentielles le long de semi-martingales en utilisant le langage du second ordre introduit par Meyer [12] et Schwartz [20]. Cette approche permet une écriture plus intrinsèque des résultats. Citons enfin le travail de Russo et Vallois [19] qui est une approche 
nouvelle et assez générale dans le cas vectoriel de l'intégration de processus quelconques le long de processus quelconques.

Nous rappellons les résultats d'Emery dans la section 0.2 ; puis, dans la section 1 , nous définissons le crochet d'une semi-martingale non-adaptée s'appuyant sur les résultats de [7] définissant les gradients stochastiques de fonctionnelles ou de processus à valeurs dans une variété.

Dans la section 2 nous définissons l'outil principal de ce calcul stochastique: il s'agit d'opérateurs de dérivation stochastique qui sont des processus à valeurs dans les vecteurs d'ordre un ou deux au-dessus de $V$. Leur existence est déduite, à l'aide du plongement de Whitney de $V$, de celle d'opérateurs de dérivation stochastique, processus à valeurs dans les vecteurs d'ordre un ou deux dans un espace vectoriel $\mathbb{R}^{p}$. Puis l'on construit les opérateurs duaux des précédents, qui permettront de définir les intégrales de 1-formes le long d'une semi-martingale non-adaptée, selon la méthode de [15]. Ces opérateurs sont un prolongement de ceux définis dans [8] lorsque le processus directeur est anticipatif au lieu d'être une semi-martingale.

Dans la section 3 nous définissons l'intégrale de 1-formes le long de processus anticipatifs à valeurs dans $V$ et nous montrons la convergence d'intégrales à variation finie vers l'intégrale de Stratonovitch de 1-formes, généralisant ainsi le lien entre la définition de Emery [4] et celle de Nualart et Pardoux [15] de ce type d'intégrale. La section 4 montre l'existence et l'unicité de la solution d'une équation différentielle stochastique, qui est un processus à valeurs dans $V$ dont la valeur initiale (et donc tout le processus) anticipe le mouvement brownien conducteur. Il est également proposé un schéma d'approximation de cette solution qui est constructif, les approximations convergeant dans $L^{r}$ uniformément sur un intervalle de temps fini.

On considère $W$ un mouvement Brownien standard de dimension $d$ défini sur son espace canonique $\left(\Omega, \mathcal{F},\left(\mathcal{F}_{t}, t \in[0 ; 1]\right), P\right)$, où $\Omega=C\left([0,1] ; \mathbb{R}^{d}\right)$ et la filtration est relative à $W$. Pour $h \in \Omega$ tel que $h$ est dans $L^{2}\left([0 ; 1], \mathbb{R}^{d}\right)$ on note $w(h)=$ $\int_{0}^{1} \dot{h}(s) d W_{s}$. $\mathcal{S}$ désigne l'ensemble des fonctionnelles de Wiener à valeurs réelles simples (Cf. Nualart [14]):

$$
\begin{aligned}
& \mathcal{S}=\left\{F \in L^{2}(\Omega) / \exists n \in \mathbb{N}, f \in C_{b}\left(\mathbb{R}^{n}\right),\right. \text { tels que: } \\
& \left.\quad F=f\left(w\left(h_{1}\right), \ldots, w\left(h_{n}\right)\right), \quad \text { avec } \quad \dot{h}_{1}, \ldots, \dot{h}_{n} \in L^{2}\left([0 ; 1] ; \mathbb{R}^{d}\right)\right\}
\end{aligned}
$$

Pour $F \in \mathcal{S}$ on définit $D F \in L^{2}\left(\Omega \times[0 ; 1] ; \mathbb{R}^{d}\right)$ par

$$
D_{t} F=\sum_{i=1}^{i=n} \frac{\partial f}{\partial x_{i}}\left(w\left(h_{1}\right), \ldots, w\left(h_{n}\right)\right) \dot{h}_{i}(t) .
$$

$D$ est le gradient stochastique usuel associé à $W$. On note, pour $p>1$, et $q \in \mathbb{N}$, $\mathbb{D}_{p, q}$ l'espace de Sobolev construit à l'aide de $D$ des fonctionnelles sur $\Omega ; \mathbb{L}_{p, q}\left(\mathbb{R}^{k}\right)$ 
est l'espace de Sobolev des fonctionnelles sur $\Omega \times[0,1]$, c'est-à-dire:

$$
\mathbb{L}_{p, q}\left(\mathbb{R}^{k}\right)=L^{p}\left([0 ; 1] ; \mathbb{D}_{p, q}\left(\mathbb{R}^{k}\right)\right)
$$

La divergence (ou intégrale de Skorokhod) $\delta$ désigne le dual de $D$. On note $\Delta$ l'opérateur Laplacien associé à $W$. Enfin, $\mathbb{L}_{2,1}^{C}\left(\mathbb{R}^{k}\right)$ désigne le sous-espace de $\mathbb{L}_{2,1}\left(\mathbb{R}^{k}\right)$ des $X$ tels que pour presque tout $t$ et pour tout $j$ les applications

$$
s \stackrel{s>t}{\longrightarrow} D_{t}^{j} X_{s} \text { et } \stackrel{s<t}{\longrightarrow} D_{t}^{j} X_{s}
$$

sont équicontinues dans $L^{2}\left(\Omega ; T \mathbb{R}^{k}\right)$, et $E\left[\left\|D_{t} X_{s}\right\|^{2}\right] \in L^{\infty}\left([0 ; 1]^{2}\right)$.

\subsection{Rappels sur l'ordre-deux}

$V$ est une variété riemannienne compacte, de classe $C^{\infty}$ et de dimension $k$ sur $\mathbb{R}$; elle est munie d'un atlas fini de cartes $\left\{\left(\varphi_{l}, U_{l}\right), l=1, \ldots, N\right\}$. L'ensemble des vecteurs tangents sur $V$ est noté $T V$; l'ensemble des 1-formes d'ordre un sur $V$ est noté $T^{*} V$ et on note ( , ) le produit de dualité entre $T V$ et $T^{*} V$. En tout point $x$ de $V$, un élément $v$ de $T_{x} V$ s'écrit $v=v^{i} \partial_{i}$ dans un système de coordonnées des dérivées partielles $\left\{\partial_{i}, i=1, \ldots, k\right\} . T_{x}^{*} V$ est engendré par les formes $\left\{d x^{i}, i=\right.$ $1, \ldots, k\}$ et, pour $\theta=\theta_{i} d x^{i}$ élément de $T_{x}^{*} V$ on a: $(\theta, v)_{x}=\theta_{i} v^{i}$.

L'ensemble des vecteurs tangents d'ordre deux sur $V$ est noté $\tau V$; c'est un fibré au-dessus de $V$, dont les fibres $\tau_{x} V$ sont engendrées, en tout point $x$ par le système de coordonnées des dérivées partielles $\left\{\partial_{i}, \partial_{i l} ; i, l=1, \ldots, k\right\}$. Si $L$ est un élément de $\tau_{x} V$, et $f$ une fonction de classe $C^{2}$ sur $V$, alors $L f(x)=v^{i l} \partial_{i l} f(x)+v^{i} \partial_{i} f(x)$, et cette écriture est unique pour un système de coordonnées si l'on impose $v^{i l}=v^{l i}$. (La convention de Einstein est ici utilisée comme elle le sera systématiquement par la suite.)

Il y a une injection canonique de $T_{x} V$ dans $\tau_{x} V$, mais la "partie d'ordre un" d'un vecteur tangent d'ordre deux $L$ n'est pas intrinsèque comme le montrent les formules de changement de coordonnées (Cf. Emery [4]):

$$
\widehat{v}^{a}=v^{i} \partial_{i} \xi^{a}+v^{i l} \partial_{i l} \xi^{a} ; \widehat{v}^{a b}=v^{i l} \partial_{i} \xi^{a} \partial_{l} \xi^{b} .
$$

où $\xi$ est l'application de changement de coordonnées.

Le fibré des formes d'ordre deux sur $V$ est noté $\tau^{*} V$, la fibre $\tau_{x}^{*} V$ est le dual topologique de $\tau_{x} V$, engendré par les formes $\left\{d^{2} x^{i}, d x^{i} \cdot d x^{l} ; i, l=1, \ldots, k\right\}$. Un élément $\theta$ de $\tau_{x}^{*} V$ s'écrit en coordonnées locales $\theta=\theta_{i} d^{2} x^{i}+\theta_{i l} d x^{i} \cdot d x^{l}$ et le changement de coordonnées s'écrit pour les formes d'ordre deux, en notant $\eta=\xi^{-1}$ :

$$
\widehat{\theta}_{a}=\partial_{a} \eta^{i} \theta_{i} ; \widehat{\theta}_{a b}=\partial_{a} \eta^{i} \partial_{b} \eta^{l} \theta_{i l}+\partial_{a b} \eta^{i} \theta_{i} .
$$

On note $\langle$,$\rangle le produit de dualité entre \tau V$ et $\tau^{*} V$; son expression en coordonnées locales est $\langle L, \theta\rangle=v^{i} \theta_{i}+v^{i l} \theta_{i l}$. 
Les formes $d^{2} f(x)$ et $d f . d g(x)$ sont définies, pour $x$ sur $V$ et pour tout $f$ et $g$ dans $C^{\infty}(V)$ :

$$
\begin{aligned}
d^{2} f(x): & L \rightarrow(L f)(x) \\
d f . d g(x): & L \rightarrow \frac{1}{2}(L(f g)(x)-f(x) L g(x)-g(x) L f(x)) .
\end{aligned}
$$

Comme on le voit par les équations (2), la partie du premier ordre d'une forme d'ordre deux $\alpha$ est intrinsèque, on peut donc définir $\mathbf{R} \alpha$, selon la notation d'Emery [4], la projection de $\alpha$ sur les formes d'ordre un.

\section{Crochet de $X$}

Soit $X$ un processus continu, mesurable à valeurs dans $V$. On note $L^{0}(\Omega \times$ $\left.[0 ; 1] ; \tau_{X} V\right)$ l'ensemble des processus à valeurs dans $\tau V$ dont la projection sur $V$ est $X$ presque sûrement. On définit de même $L^{0}\left(\Omega \times[0 ; 1] ; \tau_{X}^{*} V\right)$. D'une manière générale, on dira d'un processus $U$ à valeurs dans un fibré $B$ sur $V$ qu'il est au-dessus de $X$ si pour presque tout $(t, \omega), U(t, \omega)$ est dans $B_{X_{t}(\omega)} V$.

On se donne une métrique $b \operatorname{sur} \tau V$, c'est-à-dire une forme bilinéaire symétrique $b$ sur les vecteurs tangents d'ordre deux. Cette métrique nous permet, par dualité, de définir une métrique $b^{*}$ sur $\tau^{*} V$ par l'égalité:

$$
\forall x \in V: b_{x}^{*}\left(\xi^{*}, \xi^{\prime *}\right)=b_{x}\left(\xi, \xi^{\prime}\right)
$$

où $\xi$ est l'unique élément de $\tau_{x} V$ tel que:

$$
\forall \zeta \in \tau_{x} V:\left\langle\xi^{*}, \zeta\right\rangle_{x}=b_{x}(\xi, \zeta)
$$

On sait que pour tout $x$ de $V$ on a: $b_{x}^{*}=b_{x}^{-1}$ : la matrice inverse de $b$. On demande que $b$ restreinte à $T V$ soit $g$ la métrique riemannienne définie sur $T V$.

On définit ainsi $L^{2}\left(\Omega \times[0 ; 1] ; \tau_{X} V\right)$ et $L^{2}\left(\Omega \times[0 ; 1] ; \tau_{X}^{*} V\right)$; le premier espace est l'ensemble des processus $L=(X, v)$ à valeurs dans $\tau_{X} V$ tels que:

$$
\|L\|_{b}=\left(E \int_{0}^{1} b\left(X_{s}\right)\left(v_{s}, v_{s}\right) d s\right)^{\frac{1}{2}}<\infty
$$

et de même pour le second.

On définit aussi $L^{2}\left(\Omega \times[0 ; 1] ; T_{X} V\right)$, c'est l'espace des processus $L=(X, v)$ à valeurs dans $T_{X} V$ tels que:

$$
\|L\|_{g}=\left(E \int_{0}^{1} g\left(X_{s}\right)\left(v_{s}, v_{s}\right) d s\right)^{\frac{1}{2}}<\infty .
$$

L'espace $L^{2}\left(\Omega \times[0 ; 1] ; T_{X}^{*} V\right)$ est défini de manière analogue. 
Nous reprenons quelques notations de [7]: $V$ et $T V$ étant munis de la tribu des boréliens, on définit l'espace:

$$
\begin{aligned}
L^{2}\left(\Omega \times[0 ; 1]^{2} ; T V\right)= & \left\{G: \Omega \times[0 ; 1]^{2} \rightarrow T V,\right. \text { mesurable, } \\
& \left.E\left[\int_{0}^{1} \int_{0}^{1} g(\pi(G(s, t)))(G(s, t), G(s, t)) d s d t\right]<\infty\right\} .
\end{aligned}
$$

Si $L \in L^{2}\left(\Omega \times[0 ; 1] ; T_{X} V\right)$ alors $L \in L^{2}(\Omega \times[0 ; 1] ; T V)$ et les métriques coïncident. Dans [7] nous avons défini $\mathbb{D}_{2,1}(V)$ : c'est l'ensemble des $F$ dans $L^{2}(\Omega ; V)$ admettant un gradient stochastique. Lorsque $X$ est un processus continu, mesurable à valeurs dans $V$, tel que $X_{s}$ appartienne à $\mathbb{D}_{2,1}(V)$ pour presque tout $s$, on définit, pour tout $j=1, \ldots, d$, presque partout en $(s, t)$ et $P$ presque sûrement, le processus $D^{j} X$ de $L^{0}\left(\Omega \times[0 ; 1]^{2} ; T V\right)$ par

$$
D_{t}^{j} X_{s} \in T_{X_{s}} V ; D_{t}^{j} X_{s}(f)=D_{t}^{j}\left(f \circ X_{s}\right) \text { pour } f \in C^{\infty}(V) .
$$

Dans la dernière égalité, $D_{t}^{j}$ désigne la dérivation stochastique vectorielle usuelle.

On notera

$$
\mathbb{L}_{2,1}(V)=\left\{X / X_{t} \in \mathbb{D}_{2,1}(V) \text { et } \forall j, D^{j} X \in L^{2}\left(\Omega \times[0 ; 1]^{2} ; T V\right)\right\} .
$$

On définit de même pour $p \geq 2, \mathbb{D}_{p, 1}(V)$ comme l'ensemble des $F \in L^{p}(\Omega ; V)$ tels que $\int_{0}^{1}\left\|D_{s} F\right\|_{g(F)}^{2} d s \in L^{\frac{p}{2}}$ et $\mathbb{L}_{p, 1}(V)$ :

$$
\mathbb{L}_{p, 1}(V)=L^{p}\left([0,1], d t ; \mathbb{D}_{p, 1}(V)\right)
$$

On note $\mathbb{L}_{p, 1}^{C}(V)$ le sous-espace de $\mathbb{L}_{p, 1}(V)$ tel que pour presque tout $t$ et pour tout $j$ les applications $s \stackrel{>}{\longrightarrow} D_{t}^{j} X_{s}$ et $s \stackrel{<}{\longrightarrow} D_{t}^{j} X_{s}$ sont équicontinues dans $L^{p}(\Omega ; T V)$, et $E\left[\left\|D_{t}^{j} X_{s}\right\|_{g\left(X_{s}\right)}^{2}\right] \in L^{\infty}\left([0 ; 1]^{2}\right)$. On note enfin $\left(D_{+}^{j} X\right)_{t}=\lim _{s \rightarrow t}^{>} D_{t}^{j} X_{s}$ et $\left(D_{-}^{j} X\right)_{t}=$ $\lim _{s \rightarrow t}^{<} D_{t}^{j} X_{s}$

$\stackrel{s \rightarrow t}{\mathrm{En}}$ utilisant la projection canonique de $T V$ sur $V$, on montre facilement que pour presque tout $t,\left(D_{+}^{j} X\right)_{t}$ et $\left(D_{-}^{j} X\right)_{t}$ sont dans $L^{2}\left(\Omega ; T_{X_{t}} V\right)$ et que $D_{+}^{j} X$ et $D_{-}^{j} X$ sont dans $L^{2}\left(\Omega \times[0,1] ; T_{X} V\right)$. Notons alors

$$
\left(\nabla_{+}^{j} X\right)_{t}=\left(D_{+}^{j} X\right)_{t}+\left(D_{-}^{j} X\right)_{t} \text { et }\left(\nabla_{-}^{j} X\right)_{t}=\left(D_{+}^{j} X\right)_{t}-\left(D_{-}^{j} X\right)_{t} \text { p.p. } t
$$

Définition 1.1. Pour $X \in \mathbb{L}_{2,1}^{C}(V)$, on appelle crochet de $X$ (Cf. [6]) le vecteur d'ordre deux au-dessus de $X$ défini par:

$$
\frac{d}{d t}\{X, X\}_{t}=\sum_{j=1}^{d}\left(\left(\nabla_{+}^{j} X\right)_{t} \cdot\left(\nabla_{-}^{j} X\right)_{t}\right)
$$


Dans la définition ci-dessus, on a noté $a . b$ le vecteur d'ordre deux produit des vecteurs d'ordre un $a$ et $b$. Par la symétrie du produit $a . b$, le crochet $\frac{d}{d s}\{X, X\}$ est un opérateur symétrique.

Remarque 1.2. i/ Le crochet $\{X, X\}$ égale le crochet de variation quadratique lorsque $X$ est adapté et continu,

ii/ On peut facilement voir que $\frac{d}{d t}\{X, X\}_{t}=\sum_{j=1}^{d}\left(\left(D_{+}^{j} X\right)_{t} \cdot\left(D_{+}^{j} X\right)_{t}-\right.$ $\left.\left(D_{-}^{j} X\right)_{t} \cdot\left(D_{-}^{j} X\right)_{t}\right)$ p.s. On notera $X^{i}$ les coordonnées de $X$ dans une carte et alors on obtient que $\frac{d}{d t}\left(X^{i}, X^{l}\right)_{t}=\sum_{j=1}^{d}\left(\left(D_{+}^{j} X^{i}\right)_{t} .\left(D_{+}^{j} X^{l}\right)_{t}-\left(D_{-}^{j} X^{i}\right)_{t} .\left(D_{-}^{j} X^{l}\right)_{t}\right)$.

\section{Opérateurs de dérivation stochastique et leurs duaux}

Le processus $X$ n'est pas ici un processus adapté; on peut néanmoins dans ce cas prolonger la définition des opérateurs de dérivation stochastique et d'intégration le long de $X$ de façon tout à fait analogue à ce qui est fait dans [8]. Le théorème 2.4 prouve l'existence d'un processus dans $L^{2}\left(\Omega \times[0 ; 1] ; \tau_{X} V\right)$ qui est une dérivation stochastique le long de $X$ lorsque celui-ci est à valeurs dans une variété. Les opérateurs d'intégration, duaux des précédents, sont définis de façon habituelle. Les preuves sont omises lorsqu'elles sont semblables à celles du cas où $X$ est adapté $[8]$.

\subsection{Opérateurs de dérivation stochastique}

DÉfinition 2.1. Soit $Z$ un processus mesurable à valeurs dans $\mathbb{R}^{k}$; on dira que $Z$ est une semi-martingale anticipative réelle $(S M A R)$ si $Z \in \mathbb{L}_{2,1}^{C}\left(\mathbb{R}^{k}\right)$ et s'il existe $u \in \mathbb{L}_{2,1}\left(\mathbb{R}^{d} ; \mathbb{R}^{k}\right)$ et $z \in L^{2}\left(\Omega \times[0 ; 1] ; \mathbb{R}^{k}\right)$ tels que pour tout $t \geq 0$,

$$
Z_{t}=Z_{0}+\int_{0}^{t} u_{s} d W_{s}+\int_{0}^{t} z_{s} d s
$$

La définition est cohérente car il y a unicité de l'écriture d'un processus anticipatif sous la forme $Z_{t}=Z_{0}+\int_{0}^{t} u_{s} d W_{s}+\int_{0}^{t} z_{s} d s$ lorsque $u \in \mathbb{L}_{2,1}\left(\mathbb{R}^{d} ; \mathbb{R}^{k}\right)$. On voit que $Z$ est dans $L^{2}\left(\Omega \times[0 ; 1] ; \mathbb{R}^{k}\right)$.

On dira que $X$ est une semi-martingale anticipante à valeurs dans $V(S M A V)$ si, pour toute $f$ dans $C^{\infty}(V), f(X)$ est une $S M A R$ :

$$
f\left(X_{t}\right)=f\left(X_{0}\right)+\int_{0}^{t} u_{j}(f, s) d W_{s}^{j}+\int_{0}^{t} z(f, s) d s .
$$

Le théorème 5.2 de [15] donne une condition d'existence d'une modification continue de $Z$, condition vérifiée lorsque par exemple $u$ appartient à $\mathbb{L}_{4,1}^{C}\left(\mathbb{R}^{d} ; \mathbb{R}^{k}\right)$, ce qui sera notre cadre de travail. 
Définition 2.2. Soit $Z$ une $S M A R$ telle que $u \in \mathbb{R}_{2,1}^{C}\left(\mathbb{R}^{d} ; \mathbb{R}^{k}\right)$, soit $F \in \mathcal{S}$, $s \in[0 ; 1]$; on note $\mathbf{D}_{s}^{\tau, Z} F$ le vecteur d'ordre deux tangent en $Z_{s}$, défini par:

$$
\mathbf{D}_{s}^{\tau, Z} F=\left(u_{j}^{i}(s) D_{s}^{j} F+F z^{i}(s)\right) \partial_{i}+\frac{1}{2} F \frac{d}{d s}\left\{Z^{i}, Z^{l}\right\}_{s} \partial_{i l}
$$

pour $i, l=1, \ldots, p$ et $j=1, \ldots, d$, où la notation $\frac{d}{d s}\left\{Z^{i}, Z^{l}\right\}_{s}$ est la version vectorielle du crochet donné dans la Remarque 1.2.

Pour $F$ dans $\mathcal{S}$ et $s \in[0 ; 1]$, le vecteur d'ordre deux $\mathbf{D}_{s}^{\tau, Z} F$ s'écrit comme la somme d'un vecteur d'ordre un et d'un vecteur d'ordre deux $\mathbf{D}_{s}^{\tau, Z} F=D_{s}^{T, Z} F+$ $F L_{s}^{\tau, Z}$ où:

$$
D_{s}^{T, Z} F=u_{j}^{i}(s) D_{s}^{j} F \partial_{i} ; L_{s}^{\tau, Z}=z^{i}(s) \partial_{i}+\frac{1}{2} \frac{d}{d s}\left\{Z^{i}, Z^{l}\right\}_{s} \partial_{i l}
$$

On montre aisément que l'opérateur $D^{T, Z}: \mathcal{S} \rightarrow L^{0}\left(\Omega \times[0 ; 1] ; T_{X} \mathbb{R}^{k}\right)$ est un opérateur local, c'est-à-dire que si $A=\{\omega \in \Omega / F(\omega)=0\}$ alors $D_{s}^{T, Z} F .1_{A}=0$ p.s. puisque $D_{s}^{T, Z} F=u_{j}^{i}(s) D_{s}^{j} F \partial_{i}$ et que $D_{s}^{j} F . \mathbf{1}_{A}=0$.

Il est alors clair que $\mathbf{D}^{\tau, Z} F . \mathbf{1}_{A}=D_{s}^{T, Z} F . \mathbf{1}_{A}+F . \mathbf{1}_{A} L^{\tau, Z}=0$ p.s., donc $\mathbf{D}^{\tau, Z}$ est aussi un opérateur local.

Définition 2.3. On dira que $Z$ vérifie l'hypothèse $(\mathbf{H})$ si $Z \in \mathbb{L}_{4,1}^{C}\left(\mathbb{R}^{k}\right)$ avec $Z_{t}=Z_{0}+\int_{0}^{t} u_{s} d W_{s}+\int_{0}^{t} z_{s} d s$ et $u \in \mathbb{L}_{4,1}^{C}\left(\mathbb{R}^{d} ; \mathbb{R}^{k}\right), z \in L^{2}\left(d t ; \mathbb{R}^{k}\right)$ p.s.

On dira que la $S M A V X$ vérifie l'hypothèse $(\mathbf{H v})$ si et seulement si $\forall f \in$ $C^{\infty}(V), f(X)$ vérifie l'Hypothèse $(\mathbf{H})$.

L'hypothèse $(\mathbf{H})$ est nécessaire pour utiliser la formule d'Itô anticipative démontrée dans [17]. Cette formule mettra en évidence le caractère intrinsèque des dérivations stochastiques (Cf. le Théorème 2.4).

D'après la construction ci-dessus, on définit la différentielle d'ordre deux de $F \in \mathcal{S}$ par rapport à $Y=f(X)$, et pour $s \in[0 ; 1]$; on note $\mathbf{D}_{s}^{Y} F$ le vecteur:

$$
\mathbf{D}_{s}^{\tau, Y} F=\left(u_{j}(f, s) D_{s}^{j} F+F z(f, s)\right) \partial+\frac{1}{2} F \frac{d}{d s}\{Y, Y\}_{s} \partial^{2}
$$

pour $j=1, \ldots, d ; \partial$ est le vecteur tangent unitaire sur $\mathbb{R}$.

Pour toute fonction $f \in C^{\infty}(V ; \mathbb{R})$, on définit ainsi un vecteur d'ordre deux sur $\mathbb{R}$; de plus on peut définir un vecteur intrinsèque sur $\tau V$ :

ThÉORÈme 2.4. Soit $X$ vérifiant $\mathbf{H v}$ et $F \in \mathcal{S}$. Presque partout en $(s, \omega) \in$ $[0 ; 1] \times \Omega$, il existe un seul vecteur d'ordre deux $\mathbf{D}_{s}^{\tau, X} F(\omega)$ dans $\tau_{X_{s}(\omega)} V$ tel que pour tout $f \in C^{\infty}(V): \tau f\left(\mathbf{D}_{s}^{\tau, X} F\right)=\mathbf{D}_{s}^{\tau, Y} F$ p.s. avec $Y=f(X)$.

Preuve. On utilise le théorème de plongement de Whitney. On note $h=$ $\left(h_{1}, \ldots, h_{p}\right)$ le plongement de $V$ dans $\mathbb{R}^{p} ;$ on sait (Cf. Emery $\left.([4])\right)$ que, pour toute 
fonction $f$ de $C^{\infty}(V)$, il existe une fonction $g$ de $C^{\infty}\left(\mathbb{R}^{p}\right)$ telle que $f=g \circ h$. On note $Z=h(X)$ le processus à valeurs dans $\mathbb{R}^{p}$ image de $X$ par $h$; d'après l'hypothèse $(\mathbf{H v})$ sur $X$, le processus $D^{\tau, Z} F$ est défini pour toute fonction $F$ de $\mathcal{S}$, c'est un processus à valeurs dans $\tau_{h(X)} \mathbb{R}^{p}$.

Pour presque tout $s \in[0 ; 1]$, on note $A_{s}$ l'opérateur différentiel défini par

$$
A_{s}(f)=\mathbf{D}_{s}^{\tau, Z} F(g)
$$

où $g$ est dans $C^{\infty}\left(\mathbb{R}^{p}\right)$ tel que $f=g \circ h$. C'est-à-dire que pour tout $x$ de $V$ on a:

$$
A_{s}(x)(f)=\mathbf{D}_{s}^{\tau, Z} F(h(x))(g)
$$

L'application $g$ n'est pas unique pour $f$ donnée mais $D_{s}^{r, Z} F(g)$ est défini de manière unique car il ne dépend que du 2-jet de $g$ dans la direction de $\tau_{h(X)} h(V)$.

En effet, soit

$$
Z_{t}^{i}=Z_{0}^{i}+\int_{0}^{1} u_{j}\left(h^{i}, s\right) d W_{s}^{j}+\int_{0}^{1} z\left(h^{i}, s\right) d s
$$

soit $f \in C^{\infty}(V)$ et $g$ tel que $f=g \circ h$; la définition 2.2 donne:

$$
\begin{aligned}
\mathbf{D}_{s}^{\tau, Z} F(g)= & \left(u_{j}\left(h^{i}, s\right) D_{s}^{j} F+F z\left(h^{i}, s\right)\right) \partial_{i} g\left(h\left(X_{s}\right)\right) \\
& +\frac{1}{2} F \frac{d}{d s}\left\{h^{i}(X), h^{l}(X)\right\}_{s} \partial_{i l} g\left(h\left(X_{s}\right)\right) .
\end{aligned}
$$

Lorsque $f=g_{1} \circ h=g_{2} \circ h$ alors

$$
\partial_{l}\left(g_{1} \circ h\right)=\partial_{i}\left(g_{1}\right)(h(x)) \cdot \partial_{l} h^{i}(x)=\partial_{l}\left(g_{2} \circ h\right)=\partial_{i}\left(g_{2}\right)(h(x)) \cdot \partial_{l} h^{i}(x)
$$

et l'injectivité de $T h$ démontrée dans le lemme 2.5 ci-dessous montre que $\partial_{i}\left(g_{1}\right)(h(x))=\partial_{i}\left(g_{2}\right)(h(x))$.

En dérivant à l'ordre deux, on trouve

$$
\begin{aligned}
\partial_{k n}\left(g_{1} \circ h\right) & =\partial_{k n} g_{1}(h(x)) \cdot \partial_{i} h^{k}(x) \partial_{l} h^{n}(x)+\partial_{i} g_{1}(h(x)) \partial_{k n} h^{i}(x) \\
=\partial_{k n}\left(g_{2} \circ h\right) & =\partial_{k n} g_{2}(h(x)) \cdot \partial_{i} h^{k}(x) \partial_{l} h^{n}(x)+\partial_{i} g_{2}(h(x)) \partial_{k n} h^{i}(x)
\end{aligned}
$$

et l'injectivité de $\tau h$ montrée ci-dessous donne $\mathbf{D}_{s}^{\tau, Z} F\left(g_{1}\right)=\mathbf{D}_{s}^{\tau, Z} F\left(g_{2}\right)$ dès que $g_{1} \circ h=g_{2} \circ h$.

Lemme 2.5. Soit h le plongement de Whitney, les applications $\tau$ h et Th sont injectives (respectivement) de $\tau V$ dans $\tau \mathbb{R}^{p}$ et de $T V$ dans $T \mathbb{R}^{p}$.

Preuve. Soit $A_{1}$ et $A_{2}$ dans $\tau V$ tels que $\tau h\left(A_{1}\right)=\tau h\left(A_{2}\right)$, alors pour tout $k \in C^{\infty}\left(\mathbb{R}^{p}\right): A_{1}(k \circ h)=A_{2}(k \circ h)$. Soit $f$ dans $C^{\infty}(V)$, il existe $g \in C^{\infty}\left(\mathbb{R}^{p}\right)$ tel que $f=g \circ h$; on a

$$
A_{1}(f)=A_{1}(g \circ h)=A_{2}(g \circ h)=A_{2}(f) .
$$


Il en est de même pour $A_{1}$ et $A_{2}$ dans $T V$.

On vérifie, en utilisant le lemme (6.1) iii/ de [4] que $A$ est un processus à valeurs dans $\tau_{X} V$ :

$$
\begin{aligned}
A(x)\left(f^{3}\right) & =\mathbf{D}^{\tau, Z} F(h(x))\left(g^{3}\right) \\
& =3 g(h(x)) \mathbf{D}^{\tau, Z} F(h(x))\left(g^{2}\right)-3 g^{2}(h(x)) \mathbf{D}^{\tau, Z} F(h(x))(g)
\end{aligned}
$$

ce qui donne immédiatement (iii) pour $f$. (Il faut noter que si $f=g \circ h$ alors, $f^{2}=g^{2} \circ h$, de même pour $\left.f^{3}\right)$.

Le vecteur $A_{s}$ est noté $\mathbf{D}_{s}^{\tau, X} F$ ou $\mathbf{D}_{s}^{\tau} F$ s'il n'y a pas de confusion. Remarquons que, en prenant pour fonction $f$ les $p$ coordonnées du plongement $h$, on obtient pour tout $i: D_{s}^{\tau, X} F\left(h^{i}\right)=\left(D_{s}^{\tau, Z} F\right)^{i}$, soit exactement:

$$
\mathbf{D}^{\tau, Z} F=\tau h\left(\mathbf{D}^{\tau, X} F\right) .
$$

De plus, soit $f=g \circ h$ de $C^{\infty}(V)$, on a $\mathbf{D}^{\tau, f(X)} F=\mathbf{D}^{\tau, g(Z)} F$.

Lemme 2.6. Soit $F \in \mathcal{S}$ et $g \in C^{\infty}\left(\mathbb{R}^{p}\right)$, on a

$$
\mathbf{D}_{s}^{\tau, g(Z)} F=\tau g\left(\mathbf{D}_{s}^{\tau, Z} F\right)
$$

pour presque tout s, p.s. dans $\tau_{X_{s}} V$.

Preuve. On a

$$
\mathbf{D}_{s}^{\tau, Z} F=\left(u_{j}\left(h^{i}, s\right) D_{s}^{j} F+F z\left(h^{i}, s\right)\right) \partial_{i}+\frac{1}{2} F \frac{d}{d s}\left\{h^{i}(X), h^{l}(X)\right\}_{s} \partial_{i l} .
$$

L'hypothèse $(\mathbf{H v})$ faite sur $X$ nous permet d'appliquer la formule d'Itô anticipante vectorielle donnée par Ocone-Pardoux ([17]):

$$
g\left(Z_{t}\right)=g\left(Z_{0}\right)+\int_{0}^{1} \partial_{i} g\left(Z_{s}\right) u_{j}\left(h^{i}, s\right) d W_{s}^{j}+\int_{0}^{1} \partial_{i} g\left(Z_{s}\right) z^{i}(s) d s+\frac{1}{2}\left\{\partial_{i} g(Z), Z^{i}\right\}_{t}
$$

où $\left\{\partial_{i} g(Z), Z^{i}\right\}_{t}=\partial_{i l} g(Z)\left\{Z^{l}, Z^{i}\right\}_{t}$. Donc:

$$
\begin{aligned}
\mathbf{D}_{s}^{\tau, g(Z)} F= & {\left[\partial_{i} g\left(Z_{s}\right) u_{j}\left(h^{i}, s\right) D_{s}^{j} F+F\left(\partial_{i} g\left(Z_{s}\right) z^{i}(s)+\frac{1}{2} \frac{d}{d s}\left\{\partial_{i} g(Z), Z^{i}\right\}_{s}\right)\right] \partial } \\
& +\frac{1}{2}\left(\frac{d}{d s}\{g(Z), g(Z)\}_{s}\right) \partial^{2}
\end{aligned}
$$

D'autre part, pour tout $\varphi \in C^{\infty}(\mathbb{R})$, on a

$$
\tau g\left(\mathbf{D}_{s}^{\tau, Z} F\right)(\varphi)=\mathbf{D}_{s}^{\tau, Z} F(\varphi \circ g)
$$




$$
\begin{aligned}
= & \left(u_{j}\left(h^{i}, s\right) D_{s}^{j} F+F z\left(h^{i}, s\right)\right) \partial_{i}(\varphi \circ g) \\
& +\frac{1}{2} F \frac{d}{d s}\left\{h^{i}(X), h^{l}(X)\right\}_{s} \partial_{i l}(\varphi \circ g) \\
= & \left(u_{j}\left(h^{i}, s\right) D_{s}^{j} F+F z\left(h^{i}, s\right)\right) \varphi^{\prime}(g) . \partial_{i} g \\
& +\frac{1}{2} F \frac{d}{d s}\left\{h^{i}(X), h^{l}(X)\right\}_{s}\left[\varphi^{\prime} \partial_{i l} g+\varphi^{\prime \prime} \partial_{i} g \partial_{l} g\right] .
\end{aligned}
$$

On sait (Cf. [6]) que $\frac{d}{d s}\left\{\partial_{i} g(Z), Z^{i}\right\}_{s}=\frac{d}{d s}\left\{h^{i}(X), h^{l}(X)\right\}_{s} \partial_{i l} g$ et que $\frac{d}{d s}\{g(Z)$, $g(Z)\}_{s}=\frac{d}{d s}\left\{h^{i}(X), h^{l}(X)\right\}_{s} \partial_{i} g \partial_{l} g$; en remplaçant ces expressions dans (9) on reconnait $\mathbf{D}_{s}^{\tau, g(Z)} F(\varphi)$.

On en déduit $\mathbf{D}^{\tau, f(X)} F=\tau g \circ \tau h\left(\mathbf{D}^{\tau, X} F\right)=\tau f\left(\mathbf{D}^{\tau, X} F\right)$. Il reste à remarquer, en utilisant cette dernière égalité, que si $h^{1}$ et $h^{2}$ sont deux plongements permettant la construction de deux vecteurs tangents d'ordre deux notés $A_{1}$ and $A_{2}$, on a pour tout $f$ :

$$
A_{1}(f)=\tau f\left(A_{1}\right)=D^{\tau, f(x)} F=\tau f\left(A_{2}\right)=A_{2}(f),
$$

c'est à dire que $\mathbf{D}^{\tau, X} F$ est indépendant du plongement $h$ choisi, ce qui achève la preuve du théorème 2.4 .

Le vecteur $D_{s}^{T, X} F$ peut être défini de la même manière, c'est l'opérateur du premier ordre défini par: $f \rightarrow D_{s}^{T, Z} F(g)$. On vérifie que c'est bien un vecteur de $T_{X_{s}} V$, et on a $D_{s}^{T, Z} F=T h\left(D^{T, X} F_{s}\right)$. On note alors $L_{s}^{\tau, X} F=\mathbf{D}_{s}^{\tau, X} F-D_{s}^{T, X} F$, on en déduit que $F L_{s}^{\tau, Z}=\tau h\left(L_{s}^{\tau, X} F\right)$ et donc, puisque $\tau h$ est injective, $L_{s}^{\tau, X} F$ est de la forme $F L_{s}^{\tau, X}$, où $L_{s}^{\tau, X} \in \tau_{X_{s}} V$.

Proposition 2.7. Soit $X$ vérifiant l'hypothèse $(\mathbf{H v})$, alors:

1/ $D_{s}^{T, X} F=D_{s}^{j} F\left(\nabla_{-}^{j} X\right)_{s}$

2/ Dans une carte $\varphi$ autour de $X_{s}$ on a: $L_{s}^{\tau, X}=z\left(\varphi^{l}, s\right) \partial_{l}+\frac{1}{2} \frac{d}{d s}\left\{X^{i}, X^{l}\right\}_{s} \partial_{i l}$.

Preuve. $1 /$ Lorsque $Z$ vérifie l'hypothèse $(\mathbf{H}), D_{s}^{T, Z} F=D_{s}^{j} F\left(\nabla_{-}^{j} Z\right)_{s}$ (Cf. [15]) et $\left(\nabla_{-}^{j} X\right)_{s}$ est un vecteur d'ordre un au-dessus de $X_{s}$. D'une part $Z=h(X)$ et donc (4) montre que $\left(\nabla_{-}^{j} Z\right)_{s}=T h\left(\left(\nabla_{-}^{j} X\right)_{s}\right)$; d'autre part $D_{s}^{T, Z} F=T h\left(D_{s}^{T, X} F\right)$, donc $T h\left(D_{s}^{T, X} F\right)=T h\left(D_{s}^{j} F\left(\nabla_{-}^{j} X\right)_{s}\right)$ et, par l'injectivité de $T h, D_{s}^{T, X} F$ s'écrit $D_{s}^{j} F\left(\nabla_{-}^{j} X\right)_{s}$.

2/ On écrit $L_{s}^{\tau, Z}=z_{s}^{i} \partial_{i}+\frac{1}{2} \frac{d}{d s}\left\{h^{i}(X), h^{l}(X)\right\}_{s} \partial_{i l}$. Or $\frac{d}{d s}\{X, X\}_{s}$ est un vecteur d'ordre deux au-dessus de $X_{s}$, et $L_{s}^{\tau, Z}=\tau h\left(L_{s}^{\tau, X}\right)$; on en déduit que $z_{s}^{i}$ s'écrit sous la forme $\partial_{l} h^{i}(X) x_{s}^{l}+\frac{1}{2} \partial_{k n} h^{i}(X) \frac{d}{d s}\left\{X^{k}, X^{n}\right\}_{s}$ en notant $X^{k}$ les coordonnées de $X$ dans une carte $\varphi$ autour de $X_{s}$ et avec la notation de (5) $x_{s}^{l}=z\left(\varphi^{l}, s\right)$. Ainsi obtient-on $L_{s}^{\tau, X}=z\left(\varphi^{l}, s\right) \partial_{l}+\frac{1}{2} \frac{d}{d s}\{X, X\}_{s}$.

Remarque 2.8. L'opérateur $\mathbf{D}^{\tau, X}: \mathcal{S} \rightarrow L^{0}\left(\Omega \times[0 ; 1] ; \tau_{X} V\right)$ est un opérateur local:

$$
\tau h\left(\mathbf{D}^{\tau, X} F . \mathbf{1}_{A}\right)=\mathbf{D}^{\tau, Z} F . \mathbf{1}_{A}=0
$$


lorsque $A=\{\omega \in \Omega / F(\omega)=0\}$, puisque $\mathbf{D}^{\tau, Z}$ est local et que l'application $\tau$ h est injective. Il en est de même pour $D^{T, X}$, opérateur de $\mathcal{S}$ dans $L^{0}\left(\Omega \times[0 ; 1] ; T_{X} V\right)$.

\subsection{Opérateurs d'intégration}

Nous omettrons désormais l'exposant $X$ dans l'écriture des opérateurs de dérivation et d'intégration, nous mettrons l'exposant $Z$ quand il le faudra. On supposera que $X$ satisfait l'hypothèse (Hv).

On définit de manière habituelle les opérateurs $\delta^{\tau}$ (de domaine de définition Dom $\delta^{\tau}$ ) et $\delta^{T}$ (de domaine Dom $\delta^{T}$ ), duaux respectivement de $\mathbf{D}^{\tau}$ et $D^{T}$ :

Dom $\delta^{\tau}$ est le sous-ensemble de $L^{0}\left(\Omega \times[0 ; 1] ; \tau_{X}^{*} V\right)$ des processus $\alpha$ tels qu'il existe $c>0$ tel que pour tout $F \in \mathcal{S}$ :

$$
\left|E\left(\int_{0}^{1}\left\langle\mathbf{D}_{s}^{\tau} F, \alpha(s)\right\rangle d s\right)\right| \leq c\|F\|_{2} ;
$$

et, pour $\alpha \in \operatorname{Dom} \delta^{\tau}$ on a l'existence d'un élément unique $\delta^{\tau}(\alpha)$ dans $L^{2}(\Omega)$ tel que pour tout $F \in \mathcal{S}$ :

$$
E\left(F \delta^{\tau}(\alpha)\right)=E\left(\int_{0}^{1}\left\langle\mathbf{D}_{s}^{\tau} F, \alpha(s)\right\rangle d s\right) .
$$

De même, un processus $\theta$ de $L^{0}\left(\Omega \times[0 ; 1] ; T_{X}^{*} V\right)$ est dans Dom $\delta^{T}$ si et seulement s'il existe $c>0$ tel que pour tout $F \in \mathcal{S}$ :

$$
\left|E\left(\int_{0}^{1}\left(D_{s}^{T} F, \theta(s)\right) d s\right)\right| \leq c\|F\|_{2} ;
$$

et pour $\theta \in \operatorname{Dom} \delta^{T}, \delta^{T, Z}(\theta)$ est l'élément unique de $L^{2}(\Omega)$ tel que pour tout $F \in \mathcal{S}$ :

$$
E\left(F \delta^{T}(\theta)\right)=E\left(\int_{0}^{1}\left(D_{s}^{T} F, \theta(s)\right) d s\right)
$$

La proposition suivante donne deux exemples généraux de formes d'ordre deux qui sont dans $\operatorname{Dom} \delta^{\tau}$.

Proposition 2.9. Soit $d^{2} f$ la 1-forme d'ordre deux associée à $f$ dans $C^{\infty}(V)$, alors $\left(s \rightarrow d^{2} f\left(X_{s}\right)\right) \in \operatorname{Dom} \delta^{\tau}$ et $\delta^{\tau}\left(d^{2} f\right)=f\left(X_{1}\right)-f\left(X_{0}\right)$.

Soit $f_{1}$ aussi dans $C^{\infty}(V)$, alors la 1-forme d'ordre deux $d f . d f_{1}$ est dans Dom $\delta^{\tau}$ et on a $\delta^{\tau}\left(d f . d f_{1}\right)=\frac{1}{2}\left(\left\{f(X), f_{1}(X)\right\}_{1}-\left\{f(X), f_{1}(X)\right\}_{0}\right) ;$ où $\left\{f(X), f_{1}(X)\right\}$ est donné par $t \rightarrow T f . T f_{1}(\{X, X\})$.

Preuve. On utilisera le lemme suivant. 
Lemme 2.10. Soit $f, g$ deux fonctions de $C^{\infty}\left(\mathbb{R}^{p}\right)$ on a alors pour tout $F \in \mathcal{S}:$

$$
\begin{aligned}
& E \int_{0}^{1}\left\langle\mathbf{D}_{s}^{\tau, Z} F, d^{2} f\right\rangle_{Z_{s}} d s=E\left(F\left(f\left(Z_{1}\right)-f\left(Z_{0}\right)\right)\right) \\
& E \int_{0}^{1}\left\langle\mathbf{D}_{s}^{\tau, Z} F, d f . d g\right\rangle_{Z_{s}} d s=E\left(\frac{1}{2} F\left(\{f(Z), g(Z)\}_{1}-\{f(Z), g(Z)\}_{0}\right)\right) .
\end{aligned}
$$

Preuve. La seconde égalité est une conséquence immédiate des définitions de $\mathbf{D}_{s}^{\tau, Z} F$ et du crochet; la première est la formule d'Itô anticipative donnée par Ocone-Pardoux ([17]).

La fonction $f$ se décompose en $g \circ h$ donc (Cf. [4]) $d^{2} f=d^{2}(g \circ h)=\tau^{*} h\left(d^{2} g\right)$. On écrit alors

$$
\begin{aligned}
& E\left(\int_{0}^{1}\left\langle\mathbf{D}_{s}^{\tau} F, d^{2} f\left(X_{s}\right)\right\rangle d s\right)=E\left(\int_{0}^{1}\left\langle\mathbf{D}_{s}^{\tau} F, \tau^{*} h\left(d^{2} g\right)\left(h\left(X_{s}\right)\right)\right\rangle d s\right) \\
& =E\left(\int_{0}^{1}\left\langle\tau h\left(\mathbf{D}_{s}^{\tau} F\right), d^{2} g\left(h\left(X_{s}\right)\right)\right\rangle d s\right)=E\left(\int_{0}^{1}\left\langle\mathbf{D}_{s}^{\tau, Z} F, d^{2} g\left(Z_{s}\right)\right\rangle d s\right) \\
& =E\left(F\left(g\left(Z_{1}\right)-g\left(Z_{0}\right)\right)\right),
\end{aligned}
$$

la dernière égalité étant obtenue grâce au Lemme 2.10 ci-dessus.

En prenant le module de (12) et en utilisant que $g(Z)$ est à valeurs dans $L^{2}$, on obtient que $d^{2} f \in \operatorname{Dom} D^{\tau, X}$ et que $\delta^{\tau, X}\left(d^{2} f\right)=f\left(X_{1}\right)-f\left(X_{0}\right)$.

La deuxième partie de la proposition est immédiate même sans utiliser le lemme 2.10.

On voit ainsi que les éléments de base des formes d'ordre un et deux sont (respectivement) dans Dom $\delta^{T}$ et Dom $\delta^{\tau}$. Toute forme d'ordre deux $\alpha$ s'écrit, par une partition de l'unité, $\alpha=\sum_{m=1}^{n}\left(\alpha_{m i} d^{2} x_{m}^{i}+\alpha_{m i l} d x_{m}^{i} . d x_{m}^{l}\right)$ les propriétés des coefficients de $\alpha$ dans les bases feront que $\alpha$ sera dans Dom $\delta^{\tau}$.

La proposition suivante est l'analogue de la proposition 2.4 de [8] et se montre simplement en utilisant la proposition 2.7.

Proposition 2.11. Soit $\alpha$ dans $L^{0}\left(\Omega \times[0 ; 1] ; \tau_{X}^{*} V\right)$ et soit $\theta$ dans $L^{0}(\Omega \times$ $\left.[0 ; 1] ; T_{X}^{*} V\right)$.

1/ $\theta$ est dans $\operatorname{Dom} \delta^{T}$ si et seulement si le processus $\left(\nabla_{-} X, \theta\right)$ appartient $\grave{a}$ Dom $\delta$. Si l'une des conditions est vérifiée alors

$$
\delta^{T}(\theta)=\delta\left(\left(\nabla_{-} X, \theta\right)\right) .
$$

2/ Si $\alpha$ est tel que $\int_{0}^{1}\left\langle L_{s}^{\tau}, \alpha(s)\right\rangle d s$ est dans $L^{2}(\Omega)$ alors $\alpha$ est dans $\operatorname{Dom} \delta^{\tau}$ si et seulement si $\mathbf{R} \alpha$ est dans $\operatorname{Dom} \delta^{T}$ et, si l'une des conditions est vraie

$$
\delta^{\tau}(\alpha)=\delta\left(\left(\nabla_{-} X, \mathbf{R} \alpha\right)\right)+\int_{0}^{1}\left\langle L_{s}^{\tau}, \alpha(s)\right\rangle d s .
$$


Remarque 2.12. Les propositions 2.9 et 2.11 permettent d'écrire, pour $f$ dans $C^{\infty}(V)$ une formule d'Itô:

$$
f\left(X_{1}\right)=f\left(X_{0}\right)+\int_{0}^{1}\left(\nabla_{-}^{j} X\right)_{s}(f) d W_{s}^{j}+\int_{0}^{1} L_{s}^{\tau}(f)\left(X_{s}\right) d s
$$

on identifie ainsi $u(f, s)$ et $z(f, s)$ donnés dans la définition d'une $S M A V$ (Cf. (5)).

Nous démontrons maintenant la fermabilité des opérateurs $\mathbf{D}^{\tau, Z}$ et $D^{T, Z}$. La démonstration est analogue au cas où $X$ est adapté (Cf. [8]); elle utilise le résultat suivant (Cf. Proposition 2.6 de [8]):

LEMme 2.13. Soit $F \in \mathcal{S}, \alpha$ un processus de Dom $\delta^{\tau, Z}$ tel que $\int_{0}^{1}\left\langle L_{s}^{\tau, Z}, \alpha(s)\right\rangle d s$ est dans $L^{2}(\Omega), \theta$ un processus de Dom $\delta^{T, Z}$ alors

1/ $F \theta \in \operatorname{Dom} \delta^{T, Z}$, et $\delta^{T, Z}(F \theta)=F \delta^{T, Z}(\theta)-\int_{0}^{1}\left(D_{s}^{T, Z} F, \theta(s)\right) d s$,

2/ $F \alpha \in \operatorname{Dom} \delta^{\tau, Z}$ et $\delta^{\tau, Z}(F \alpha)=F \delta^{\tau, Z}(\alpha)-\int_{0}^{1}\left(D_{s}^{T, Z} F, \mathbf{R} \alpha(s)\right) d s$.

Ainsi, formellement, la même démonstration que celle de la proposition 1.5 de [8] montre:

Proposition 2.14. Si $Z$ vérifie l'hypothèse $(\mathbf{H})$ les opérateurs $\mathbf{D}^{\tau, Z}$ et $D^{T, Z}$ sont fermables en tant qu'opérateurs non bornés de $\mathcal{S}$ dans respectivement $L^{2}(\Omega \times$ $\left.[0 ; 1] ; \tau_{Z} h(V)\right)$ et $L^{2}\left(\Omega \times[0 ; 1] ; T_{Z} h(V)\right)$.

Ce qui donne en corollaire la fermabilité des opérateurs $\mathbf{D}^{\tau}$ et $D^{T}$ :

Proposition 2.15. Si $X$ vérifie l'hypothèse $(\mathbf{H v})$ les opérateurs $\mathbf{D}^{\tau}$ et $D^{T}$ sont fermables en tant qu'opérateurs non bornés de $\mathcal{S}$ dans respectivement $L^{2}(\Omega \times$ $\left.[0 ; 1] ; \tau_{X} V\right)$ et $L^{2}\left(\Omega \times[0 ; 1] ; T_{X} V\right)$.

Preuve. On se donne une suite $\left(F_{n}\right)_{n}$ de fonctions dans $\mathcal{S}$ telles que $F_{n} \rightarrow F$ et $\mathbf{D}^{\tau} F_{n} \rightarrow G$, alors $\tau h\left(\mathbf{D}^{\tau} F_{n}\right) \rightarrow \tau h(G)$. La proposition 2.14 donne que $\mathbf{D}^{\tau, Z}$ est fermable donc $F$ est $\mathbf{D}^{\tau, Z}$-dérivable et $\mathbf{D}^{\tau, Z} F=\tau h(G)$, d'où, par l'injectivité de $\tau h, F$ est $\mathbf{D}^{\tau}$-dérivable et $\mathbf{D}^{\tau} F=G$.

Pour $p>1$, on note $\mathbb{D}_{p, 1}^{\tau}$ la fermeture de $\mathcal{S}$ selon la norme $\left(\|F\|_{p}^{p}+\left\|\mathbf{D}^{\tau} F\right\|_{b}^{p}\right)^{\frac{1}{p}}$ et $\mathbb{D}_{p, 1}^{T}$ la fermeture de $\mathcal{S}$ selon la norme $\left(\|F\|_{p}^{p}+\left\|D^{T} F\right\|_{g}^{p}\right)^{\frac{1}{p}}$. On déduit alors immédiatement (Cf. La proposition 2.7 de [8]):

Proposition 2.16. Soit $p>1$ et $F \in \mathbb{D}_{p, 1}^{T}, \alpha \in \operatorname{Dom} \delta^{\tau}, \theta \in \operatorname{Dom} \delta^{T}$ alors,

1/ Si $F \delta^{\tau}(\alpha)-\int_{0}^{1}\left(D_{s}^{T} F, \mathbf{R} \alpha(s)\right) d s \in L^{2}(\Omega)$ on $a: F \alpha \in \operatorname{Dom} \delta^{\tau}$ et $\delta^{\tau}(F \alpha)$ est donné par l'expression ci-dessus.

2/ Si $F \delta^{T}(\theta)-\int_{0}^{1}\left(D_{s}^{T} F, \theta(s)\right) d s \in L^{2}(\Omega)$ on a: $F \theta \in \operatorname{Dom} \delta^{T}$ et $\delta^{T}(F \theta)$ est donné par l'expression ci-dessus. 


\section{Intégrales de 1-formes et Approximations}

Dans cette section, lorsque nous parlerons de processus à valeurs 1-formes, il s'agira toujours de processus à valeurs dans les champs $C^{\infty}$ de formes d'ordre un sur $V$ (les processus à valeurs dans les formes d'ordre un ou deux au-dessus de $X$ ne sont pas a priori des champs.)

\subsection{Intégrale de Stratonovitch des 1-formes}

Pour $\alpha$ une 1-forme (champ) décrite localement par $\alpha_{i} d x^{i}$ Meyer [12] définit un processus $d \alpha$ a valeurs dans $\tau_{X}^{*} V$ :

$$
d \alpha(s)=\alpha_{i}\left(X_{s}\right) d^{2} x^{i}+\partial_{i} \alpha_{l}\left(X_{s}\right) d x^{i} \cdot d x^{l} .
$$

DÉfinition 3.1. Soit $\alpha \in \mathcal{X}^{*}(V)$ tel que $d \alpha$ soit dans Dom $\delta^{\tau}$; on appelle intégrale de Stratonovitch de $\alpha$ :

$$
S^{\tau, X}(\alpha)=\delta^{\tau}(d \alpha)
$$

Pour que $d \alpha$ soit dans Dom $\delta^{\tau}$ il suffit que $\alpha(=\mathbf{R}(d \alpha))$ appartienne à Dom $\delta^{T}$ et que $\int_{0}^{1}\left\langle L_{s}^{\tau}, d \alpha(s)\right\rangle d s$ appartienne à $L^{2}(\Omega)$. Dans ce cas,

$$
S^{\tau}(\alpha)=\delta^{T}(\alpha)+\int_{0}^{1}\left\langle L_{s}^{\tau}, d \alpha(s)\right\rangle d s
$$

Proposition 3.2. Si $\alpha \in \mathcal{X}^{*}(V)$, et si $X$ est une $S M A V$ vérifiant les hypothèses $(\mathbf{H v})$, alors

$$
S^{\tau, X}(\alpha)=\delta\left(\left(\nabla_{-} X, \alpha\right)\right)+\int_{0}^{1} z\left(\varphi^{i}, s\right) \alpha_{i}\left(X_{s}\right) d s+\frac{1}{2} \int_{0}^{1}\left(\nabla_{+}^{j}(\alpha(X))\right)_{s}\left(\nabla_{-}^{j} X\right)_{s} d s .
$$

Preuve. Il suffit de remarquer que localement $\left(\nabla_{+}^{j}(\alpha(X))\right)_{s}$ s'écrit $\partial_{i} \alpha_{l}\left(X_{s}\right)$ $\left(\nabla_{+}^{j} X^{i}\right)_{s}$. Par ailleurs,

$$
\left\langle L_{s}^{\tau, X}, d \alpha\right\rangle=z\left(\phi^{i}, s\right) \alpha_{i}\left(X_{s}\right)+\frac{1}{2} \partial_{i} \alpha_{l}\left(X_{s}\right)\left(\nabla_{+}^{m} X^{i}\right)_{s}\left(\nabla_{-}^{m} X^{l}\right)_{s} .
$$

On obtient le résultat.

Lemme 3.3. Soit $\alpha \in \mathcal{X}^{*}(V)$ et $\widetilde{\alpha}$ tel que $(T h)^{*}(\widetilde{\alpha})=\alpha$. La forme d'ordre

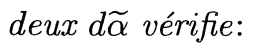

$$
(\tau h)^{*}(d \widetilde{\alpha})=d \alpha .
$$


Preuve. Les définitions de ces deux formes d'ordre deux permettent leur identification dans des cartes locales.

Corollaire 3.4. Soit $\alpha \in \mathcal{X}^{*}(V)$ et $\widetilde{\alpha}$ tel que $(T h)^{*}(\widetilde{\alpha})=\alpha$. Alors:

$$
S^{\tau, X}(\alpha)=S^{\tau, Z}(\widetilde{\alpha})
$$

La preuve est immédiate puisque

$$
S^{\tau, X}(\alpha)=\delta^{\tau, X}(d \alpha)=\delta^{\tau, Z}(d \widetilde{\alpha})=S^{\tau, Z}(\widetilde{\alpha}),
$$

la deuxième égalité étant l'équation duale de (7).

Remarque 3.5. L'intégrale de Stratonovitch ainsi définie ne vérifie pas la propriété habituelle $S^{\tau}(F \alpha)=F S^{\tau}(\alpha)$ pour $F \in \mathcal{S}$; il faudrait pour cela définir l'intégrale de Stratonovitch d'un champ aléatoire de 1-formes. C'est possible en supposant l'hypothèse $(\mathbf{I})$ : presque partout en $s$, l'opérateur $\frac{d}{d s}\{X, X\}_{s}$ à valeurs dans $\tau_{X} V$ est de rang plein. On peut alors définir une opération $\alpha \rightarrow d_{X} \alpha$ qui généralise aux 1-formes aléatoires l'opération $\alpha \rightarrow d \alpha$ : Pour un $p \geq 2, \alpha$ un élément de $\mathbb{L}_{p, 1}\left(\mathcal{X}^{*}(V)\right)$ (c'est à dire que $\omega \mapsto \alpha(\omega, X(\omega)) \in \mathbb{L}_{p, 1}\left(\tau_{X}^{*}(V)\right)$ ), alors, la forme d'ordre deux $d_{X} \alpha$ est définie localement pour tout $s$ par:

$$
\begin{aligned}
\left(d_{X} \alpha\right)_{i}(s) & =\alpha_{i}\left(X_{s}\right) \\
\left(d_{X} \alpha\right)_{i l}(s) & =\partial_{i} \alpha_{l}\left(X_{s}\right)+2\left[\left(\left(\nabla_{+}^{j} X\right)_{s}\left(\nabla_{-}^{j} X\right)_{s}\right)^{-1}\left(\nabla_{-}^{m} X\right)_{s}\right]_{i}\left(\nabla_{+}^{m} \alpha_{l}\right)_{s} .
\end{aligned}
$$

On démontre (Cf. [8]) que la définition est intrinsèque.

La proposition 3.2 est encore vraie pour $\alpha \in \mathbb{L}_{p, 1}\left(\mathcal{X}^{*}(V)\right)$, lorsque $X$ vérifie l'hypothèse $(\mathbf{I})$; en effet, il suffit de remarquer que localement $\left(\nabla_{+}^{j}(\alpha(X))\right)_{s}$ s'écrit $\partial_{i} \alpha_{l}\left(X_{s}\right)\left(\nabla_{+}^{j} X^{i}\right)_{s}+\left(\nabla_{+}^{j} \alpha_{l}\right)_{s}\left(X_{s}\right)$, la deuxième dérivation stochastique étant entendue uniquement sur la part aléatoire de $\alpha(\omega, x)$.

\subsection{Approximation de l'intégrale de Stratonovitch}

On démontre un théorème d'approximation de l'intégrale de Stratonovitch d'une 1-forme le long de la semi-martingale anticipante $X$ s'appuyant sur une interpolation $I$ sur $V$ ([4] p.14), à savoir:

$$
I: V \times V \times[0,1] \rightarrow V
$$

telle que pour tour $(x, y)$ de $V \times V, I(x, y, \cdot)$ est une courbe régulière de $x$ à $y$ vérifiant uniformément sur tout compact:

$$
\frac{\partial^{m}}{\partial t^{m}} I(x, y, t)=O\left[d^{m}(x, y)\right], \quad m=1,2,3 .
$$


On démontre ainsi que si $d \alpha$ est dans Dom $\delta^{\tau}$ alors $\alpha$ est "Stratonovitch intégrable" au sens de $[15]$.

Soit $\sigma=\left\{0=T_{1}, \ldots, T_{n}=1\right\}$ une subdivision de $[0,1]$ dont le pas $\sup _{l}\left|T_{l+1}-T_{l}\right|$ tend vers zéro quand $n$ tend vers l'infini; on note $\Delta_{l} T=\left|T_{l+1}-T_{l}\right|$. Soit $X^{n}$ le processus approchant $X$ sur $V$ :

$$
X_{t}^{n}=I\left(X_{T_{l}}, X_{T_{l+1}}, \frac{t-T_{l}}{\Delta_{l} T}\right) \text { pour } t \in\left[T_{l}, T_{l+1}\right] \text { et } l=1, \ldots, n .
$$

Utilisant le lemme (7.26) de [4], et le plongement de Whitney $h$ de $V$ dans $\mathbb{R}^{p}$, on a l'existence d'une interpolation $J$ sur $\mathbb{R}^{p}$ telle que:

$$
J(h(x), h(y), t)=h \circ I(x, y, t) .
$$

On note alors $Z^{n}$ le processus vectoriel défini par:

$$
Z_{t}^{n}=h\left(X^{n}\right)=J\left(Z_{T_{l}}, Z_{T_{l+1}}, \frac{t-T_{l}}{\Delta_{l} T}\right) \text { pour } t \in\left[T_{l}, T_{l+1}\right] \text { et } l=1, \ldots, n .
$$

ThÉORÈme 3.6. Soit $\alpha \in \mathcal{X}^{*}(V)$, alors,

$$
\lim _{\text {proba }} \rightarrow \infty \int_{0}^{1}\left(\alpha, \dot{X}^{n}\right)_{s} d s=S^{\tau, X}(\alpha) .
$$

Preuve. Du fait que $Z^{n}=h\left(X^{n}\right), \dot{Z}^{n}=T h\left(\dot{X}^{n}\right)$ et $\left(\alpha, \dot{X}^{n}\right)_{s}=$ $\left((T h)^{*}(\alpha), \dot{Z}^{n}\right)$ que l'on note $\left(\widetilde{\alpha}, \dot{Z}^{n}\right)$ :

$$
\int_{0}^{1}\left(\alpha, \dot{X}^{n}\right)_{s} d s=\int_{0}^{1}\left(\widetilde{\alpha}, \dot{Z}^{n}\right)_{s} d s
$$

On pose $a(u)=T_{l}+u \Delta_{l} T, u \in[0,1]$; soit:

$$
Z_{a(u)}^{n}=J\left(Z_{T_{l}}, Z_{T_{l+1}}, u\right) .
$$

On pose pour tout $u \in[0,1]$ :

$$
Y_{a(u)}^{n}-Y_{T_{l}}^{n}=\int_{0}^{1}\left(\widetilde{\alpha}_{i}\left(Z_{a}^{n}(v)\right), \dot{Z}_{a(v)}^{n, i}\right) d v
$$

Utilisant directement les calculs de Emery [4] p.101, à savoir

$$
\dot{J}\left(Z_{T_{l}}, Z_{T_{l+1}}, v\right)=\Delta_{l} Z+\left(v-\frac{1}{2}\right) \ddot{J}\left(Z_{T_{l}}, Z_{T_{l+1}}, 0\right)+O\left(\left\|\Delta_{l} Z\right\|^{3}\right)
$$


et un développement limité de $\widetilde{\alpha}_{i}$ en $Z_{T_{l}}$ :

$$
\widetilde{\alpha}_{i}\left(Z_{a(v)}^{n}\right)=\widetilde{\alpha}_{i}\left(Z_{T_{l}}^{n}\right)+\partial_{k} \widetilde{\alpha}_{i}\left(Z_{T_{l}}\right)\left(Z_{a(v)}^{n, k}-Z_{T_{l}}^{k}\right)+O\left(\left\|\Delta_{l} Z\right\|^{2}\right)
$$

on obtient une approximation de (19) à l'ordre 3:

$$
\begin{aligned}
Y_{a(u)}^{n}-Y_{T_{l}}^{n}= & \int_{0}^{u}\left[\widetilde{\alpha}_{i}\left(Z_{T_{l}}\right)+\partial_{k} \widetilde{\alpha}_{i}\left(Z_{T_{l}}\right)\left(Z_{a(v)}^{n, k}-Z_{T_{l}}^{k}\right)\right] \Delta_{l} Z^{i} d v \\
& +\int_{0}^{u} \widetilde{\alpha}_{i}\left(Z_{T_{l}}\right)\left(v-\frac{1}{2}\right) \ddot{J}\left(Z_{T_{l}}, Z_{T_{l+1}}, 0\right) d v+O\left(\left\|\Delta_{l} Z\right\|^{3}\right) .
\end{aligned}
$$

Réutilisant (20), on obtient:

$$
Z_{a(v)}^{n}-Z_{T_{l}}=\int_{O}^{v} \dot{J}\left(Z_{T_{l}}, Z_{T_{l+1}}, s\right) d s=v \cdot \Delta_{l} Z+O\left(\left\|\Delta_{l} Z\right\|^{2}\right)
$$

et puisque $\int_{0}^{1}\left(v-\frac{1}{2}\right) d v=0$, il vient pour $u=1$ :

$$
Y_{T_{l+1}}^{n}-Y_{T_{l}}^{n}=\widetilde{\alpha}_{i}\left(Z_{T_{i}}\right) \Delta_{l} Z^{i}+\frac{1}{2} \partial_{k} \widetilde{\alpha}_{i}\left(Z_{T_{l}}\right) \Delta_{l} Z^{k} \Delta_{l} Z^{i}+O\left(\left\|\Delta_{l} Z\right\|^{3}\right) .
$$

Or, $\Delta_{l} Z^{i}=\int_{I_{l}}\left(\nabla_{-}^{j} Z^{i}\right)_{s} d W_{s}^{j}+z^{i}(s) d s$. Le produit des accroissements de $Z$ se développe en:

$$
\begin{aligned}
\Delta_{l} Z^{i} \Delta_{l} Z^{k}= & \int_{I_{l}}\left(\Delta_{-}^{j} Z^{i}\right)_{s} d W_{s}^{j} \int_{I_{l}}\left(\nabla_{-}^{j} Z^{k}\right)_{s} d W_{s}^{j}+\int_{I_{l}} z^{i}(s) d s \int_{I_{l}} z^{k}(s) d s \\
& +\int_{I_{l}}\left(\nabla_{-}^{j} Z^{i}\right)_{s} d W_{s}^{j} \int_{I_{l}} z^{k}(s) d s+\int_{I_{l}}\left(\nabla_{-}^{j} Z^{k}\right)_{s} d W_{s}^{j} \int_{I_{l}} z^{i}(s) d s
\end{aligned}
$$

Les hypothèses sur $X$, donc sur $Z$, montrent que les processus $\left(\nabla_{-}^{j} Z^{i}\right)_{s}$ appartiennent à $\mathbb{L}_{4,1}$ et le théorème 5.4 de [15] montre que la somme des premiers termes de (23) converge en probabilité:

$$
\lim \operatorname{proba}_{n \rightarrow \infty} \sum_{l=1}^{n} \int_{I_{l}}\left(\nabla_{-}^{j} Z^{i}\right)_{s} d W_{s}^{j} \int_{I_{l}}\left(\nabla_{-}^{j} Z^{k}\right)_{s} d W_{s}^{j}=\int_{0}^{1}\left(\nabla_{-}^{j} Z^{i}\right)_{s}\left(\nabla_{-}^{j} Z^{k}\right)_{s} d s .
$$

La somme des deuxièmes termes de (23) se majore par:

$$
\left|\sum_{l} \int_{I_{l}} z^{i}(s) d s \int_{I_{l}} z^{k}(s) d s\right| \leq \sup _{l} \int_{I_{l}}\left|z^{i}(s)\right| d s \int_{0}^{1}\left|z^{k}(s)\right| d s
$$

qui converge presque sûrement vers 0 puisque le processus $z$ est intégrable sur $[0,1]$. 
Les derniers termes se majorent en norme $L^{1}$ :

$$
\left\|\int_{I_{l}}\left(\nabla_{-}^{j} Z^{i}\right)_{s} d W_{s}^{j} \int_{I_{l}} z^{k}(s) d s\right\|_{1} \leq\left\|\int_{I_{l}}\left(\nabla_{-}^{j} Z^{i}\right)_{s} d W_{s}^{j}\right\|_{2}\left\|\int_{I_{l}} z^{k}(s) d s\right\|_{2}
$$

dont la somme est majorée par:

$$
\sup _{l}\left\|\int_{I_{l}}\left(\nabla_{-}^{j} Z^{i}\right) s d W_{s}^{j}\right\|_{2} \sum_{l}\left\|\int_{I_{l}} z^{k}(s) d s\right\|_{2} .
$$

Le deuxième facteur ci-dessus est borné puisque $z \in L^{2}(\Omega \times[0,1])$ et puisqu'il est clair que $\left(\nabla_{-}^{j} Z^{i}\right)_{s} 1_{I_{l}}$ converge vers zéro dans $\mathbb{L}_{2,1}$, son intégrale de Skohorod tend vers zéro dans $L^{2}(\Omega)$ et la limite de $(24)$ est nulle. En résumé,

$$
\lim \operatorname{proba}_{n \rightarrow \infty} \sum_{l=1}^{n} \Delta_{l} Z^{i} \Delta_{l} Z^{k}=\int_{0}^{1}\left(\nabla_{-}^{j} Z^{i}\right)_{s}\left(\nabla_{-}^{j} Z^{k}\right)_{s} d s
$$

Puisque $\alpha$, donc $\widetilde{\alpha}$, est à dérivées bornées et que $Z$ est continue:

$$
\lim \operatorname{proba}_{n \rightarrow \infty} \sum_{l=1}^{n} \partial_{k} \widetilde{\alpha}_{i}\left(Z_{T_{l}}\right) \Delta_{l} Z^{i} \Delta_{l} Z^{k}=\int_{0}^{1} \partial_{k} \widetilde{\alpha}_{i}\left(Z_{s}\right)\left(\nabla_{-}^{j} Z^{i}\right)_{s}\left(\nabla_{-}^{j} Z^{k}\right)_{s} d s .
$$

On récrit maintenant le premier terme de (22) avec la formule d'intégration par parties:

$$
\begin{aligned}
\widetilde{\alpha}_{i}\left(Z_{T_{l}}\right) \Delta_{l} Z^{i}= & \int_{I_{l}} \widetilde{\alpha}_{i}\left(Z_{T_{l}}\right)\left[\left(\nabla_{-}^{j} Z^{i}\right)_{s} d W_{s}^{j}+z^{i}(s) d s\right] \\
& +\int_{I_{l}} D_{s}^{j}\left(\widetilde{\alpha}_{i}\left(Z_{T_{l}}\right)\right)\left(\nabla_{-}^{j} Z^{i}\right)_{s} d s .
\end{aligned}
$$

On pose alors $u_{s}^{n}=\left(\widetilde{\alpha}_{i}\left(Z_{s}\right)-\widetilde{\alpha}_{i}\left(Z_{T_{l}}\right)\right)\left(\nabla_{-}^{j} Z^{i}\right)_{s}$. Let hypothèses de régularité sur $Z$ (hypothèse $(\mathbf{H}))$ et sur $\alpha$, le fait que $\widetilde{\alpha}$ et $\partial_{k} \widetilde{\alpha}$ soient continues et bornées, montrent que la suite $\left(u^{n}\right)$ converge vers zéro dans $\mathbb{L}_{2,1}$, puisque

$$
\begin{aligned}
D_{t} u_{s}^{n}= & \left(\partial_{k} \widetilde{\alpha}_{i}\left(Z_{s}\right) D_{t} Z_{s}^{k}-\partial_{k} \widetilde{\alpha}_{i}\left(Z_{T_{l}}\right) D_{t} Z_{T_{l}}^{k}\right)\left(\nabla_{-}^{j} Z^{i}\right)_{s} \\
& +\left(\widetilde{\alpha}_{i}\left(Z_{s}\right)-\widetilde{\alpha}_{i}\left(Z_{T_{l}}\right)\right) D_{t}\left(\nabla_{-}^{j} Z^{i}\right)_{s} .
\end{aligned}
$$

On a ainsi la convergence dans $L^{2}$ donc en probabilité, de la somme des premiers termes de (26):

$$
\lim _{n \rightarrow \infty} \sum_{l=1}^{n} \int_{I_{l}} \widetilde{\alpha}_{i}\left(Z_{T_{l}}\right)\left(\nabla_{-}^{j} Z^{i}\right)_{s} d W_{s}^{j}=\int_{0}^{1} \widetilde{\alpha}_{i}\left(Z_{s}\right)\left(\nabla_{-}^{j} Z^{i}\right)_{s} d W_{s}^{j}
$$


Enfin, on peut expliciter le reste de (26):

$$
\begin{aligned}
\int_{I_{l}} & {\left[\widetilde{\alpha}_{i}\left(Z_{T_{l}}\right) z^{i}(s)+D_{s}^{j}\left(\widetilde{\alpha}_{i}\left(Z_{T_{l}}\right)\right)\left(\nabla_{-}^{j} Z^{i}\right)_{s}\right] d s } \\
= & \int_{I_{l}}\left[\widetilde{\alpha}_{i}\left(Z_{T_{l}}\right) z^{i}(s)+\partial_{k} \widetilde{\alpha}_{i}\left(Z_{T_{l}}\right) D_{t}^{j} Z_{T_{l}}^{k}\left(\nabla_{-}^{j} Z^{i}\right)_{s}\right] d s .
\end{aligned}
$$

Par continuité de $\widetilde{\alpha}$ et de $\partial \widetilde{\alpha}$ et par définition de l'opérateur $D_{-}$, l'intégrand converge presque sûrement vers:

$$
\widetilde{\alpha}_{i}\left(Z_{s}\right) z^{i}(s)+\partial_{k} \widetilde{\alpha}_{i}\left(Z_{s}\right)\left(D_{-}^{j} Z^{k}\right)_{s}\left(\nabla_{-}^{j} Z^{i}\right)_{s} .
$$

Soit:

$$
\begin{gathered}
\lim \operatorname{proba}_{n \rightarrow \infty} \sum_{l=1}^{n} \int_{I_{l}}\left[\widetilde{\alpha}_{i}\left(Z_{T_{l}}\right) z^{i}(s)+D_{s}^{j}\left(\widetilde{\alpha}_{i}\left(Z_{T_{l}}\right)\right)\left(\nabla_{-}^{j} Z^{i}\right)_{s}\right] d s \\
=\int_{0}^{1}\left[\widetilde{\alpha}_{i}\left(Z_{s}\right) z^{i}(s)+\partial_{k} \widetilde{\alpha}_{i}\left(Z_{s}\right)\left(D_{-}^{j} Z^{k}\right)_{s}\left(\nabla_{-}^{j} Z^{i}\right)_{s}\right] d s .
\end{gathered}
$$

Notons enfin:

$$
E_{l}^{n}=Y_{T_{l+1}}^{n}-Y_{T_{l}}^{n}-\left[\widetilde{\alpha}_{i}\left(Z_{T_{l}}\right) \Delta_{l} Z^{i}+\frac{1}{2} \partial_{k} \widetilde{\alpha}_{i}\left(Z_{T_{l}}\right) \Delta_{l} Z^{i} \Delta_{l} Z^{k}\right] .
$$

On a montré en $(22)$ que $\left\|E_{l}^{n}\right\|=O\left(\left\|\Delta_{l} Z\right\|^{3}\right)$. Or,

$$
\sum_{l=1}^{n}\left\|\Delta_{l} Z\right\|^{3} \leq \sup _{l}\left\|\Delta_{l} Z\right\| \sum_{l}\left\|\Delta_{l} Z\right\|^{2} .
$$

Le premier facteur tend par continuité presque sûrement vers 0 . La somme est presque sûrement finie puisque $\Delta_{l} Z=\delta\left(u 1_{I_{l}}\right)+\int_{I_{l}} z(s) d s$. Alors,

$$
\begin{aligned}
E\left(\Delta_{l} Z\right)^{2} & \leq 2 E\left[\delta\left(u 1_{I_{l}}\right)^{2}\right]+2 E\left\|\int_{I_{l}} z(s) d s\right\|^{2} \\
& \leq 2 \int_{I_{l}}\|u(s)\|^{2} d s+2 \int_{I_{l}} \int_{I_{l}} E\left[D_{s} u_{t} D_{t} u_{s}\right] d s d t+2 \Delta_{l} T \int_{I_{l}}\|z(s)\|^{2} d s
\end{aligned}
$$

dont la somme est évidemment finie puisque $u \in \mathbb{L}_{4,1}$ et $z \in L^{2}(\Omega \times[0,1])$.

Ce dernier résultat joint à (25), (27) et (29) montre que:

$$
\begin{aligned}
& \lim \operatorname{proba}_{n \rightarrow \infty}\left(Y_{1}^{n}-Y_{0}^{n}\right) \\
& =\int_{0}^{1} \widetilde{\alpha}_{i}\left(Z_{s}\right)\left[\left(\nabla_{-}^{j} Z^{i}\right)_{s} d W_{s}^{j}+z^{i}(s) d s\right] \\
& \quad+\int_{0}^{1} \partial_{k} \widetilde{\alpha}_{i}\left(Z_{s}\right)\left[\frac{1}{2}\left(\nabla_{-}^{j} Z^{k}\right)_{s}\left(\nabla_{-}^{j} Z^{i}\right)_{s}+\left(D_{-}^{j} Z^{k}\right)_{s}\left(\nabla_{-}^{j} Z^{i}\right)_{s}\right] d s .
\end{aligned}
$$


Or $\frac{1}{2}\left(\nabla_{-}^{j} Z^{k}\right)+\left(D_{-}^{j} Z^{k}\right)=\nabla_{+}^{j} Z^{k}$. On reconnait dans cette limite l'intégrale de $d \widetilde{\alpha}$ le long de la semi-martingale $Z$, ce qui achève de montrer la proposition avec l'égalité (18) et le corollaire 3.4 .

Remarque 3.7. Considérons sur $V$ sa connexion sans torsion $\Gamma$. Meyer ([12]) définit, pour $\alpha$ un processus prévisible de $L^{0}\left(\Omega \times[0,1] ; T_{X}^{*} V\right)$, défini localement par $\alpha_{i}(s) d x^{i}$, un processus $\Gamma^{*} \alpha$ à valeurs dans $\tau_{X}^{*} V$ :

$$
\Gamma^{*} \alpha(s)=\alpha_{i}(s) d^{2} x^{i}+\Gamma_{l m}^{i}\left(X_{s}\right) \alpha_{i}(s) d x^{l} \cdot d x^{m} .
$$

Nous pouvons étendre cette définition au cas où la semi-martingale $X$ n'est plus adaptée: soit $\alpha$ un processus à valeurs dans $L^{2}\left(\Omega \times[0,1] ; T_{X}^{*} V\right)$ tel que $\Gamma^{*} \alpha$ soit dans Dom $\delta^{\tau}$; on note $I^{\tau}(\alpha)$ l'intégrale d'Itô de $\alpha$, définie par:

$$
I^{\tau}(\alpha)=\delta^{\tau}\left(\Gamma^{*} \alpha\right) \text {. }
$$

Pour que $\Gamma^{*} \alpha$ soit dans Dom $\delta^{\tau}$ il suffit que $\alpha\left(=\mathbf{R}\left(\Gamma^{*} \alpha\right)\right)$ appartienne à Dom $\delta^{T}$ et que $\int_{0}^{1}\left\langle L_{s}^{\tau}, \Gamma^{*} \alpha(s)\right\rangle d s$ appartienne à $L^{2}(\Omega)$. Dans ce cas,

$$
I^{\tau}(\alpha)=\delta^{T}(\alpha)+\int_{0}^{1}\left\langle L_{s}^{\tau}, \Gamma^{*} \alpha(s)\right\rangle d s
$$

Enfin, $\left(I^{\tau}\right)^{*}=\Gamma \circ \mathbf{D}^{\tau}$ est un opérateur qui associe à tout $F$ de $\mathcal{S}$ un processus à valeurs dans $T V . \Gamma \circ \mathbf{D}_{s}^{\tau} F$ est un vecteur tangent en $X_{s}$, projection horizontale de $\mathbf{D}_{s}^{\tau} F$ selon la connexion $\Gamma$ (Cf. [7]); c'est une dérivée stochastique covariante de $F$ par rapport à $X$ (Cf. [13]). Fang et Malliavin dans [5] définissent des opérateurs tout à fait analogues, mais ils évitent le calcul d'ordre deux en se servant d'outils tels le fibré des repères et le transport parallèle "stochastique", mais leurs opérateurs sont relatifs à un processus sous-jacent adapté.

\section{E.D.S. et Approximations}

On note $\mathbb{L}_{p, 1}\left(T_{X}^{*} V\right)$ le sous-espace de $L^{0}\left(\Omega \times[0 ; 1] ; T_{X}^{*} V\right)$ des processus $\theta$ tels que $\left(\nabla_{-} X, \theta\right)$ est dans $\mathbb{L}_{p, 1}$, et $\mathbb{L}_{p, 1}\left(\tau_{X}^{*} V\right)$ le sous-espace de $L^{0}\left(\Omega \times[0 ; 1] ; \tau_{X}^{*} V\right)$ des processus $\alpha$, tels que $\mathbf{R} \alpha$ est dans $\mathbb{L}_{p, 1}\left(T_{X}^{*} V\right)$ et $\int_{0}^{1}\left\langle L_{s}^{\tau}, \alpha(s)\right\rangle d s$ dans $L^{p \vee 2}(\Omega)$.

Proposition 4.1. $\quad \mathbb{L}_{p, 1}\left(T_{X}^{*} V\right)$ est inclus dans $\operatorname{Dom} \delta^{T}$, et $\mathbb{L}_{p, 1}\left(\tau_{X}^{*} V\right)$ est inclus dans $\operatorname{Dom} \delta^{\tau}$.

Preuve. On montre la première inclusion, la seconde s'en déduit. Soit $\theta$ dans $\mathbb{L}_{p, 1}\left(T_{X}^{*} V\right)$, alors $\left(\nabla_{-} X, \theta\right)$ est dans $\mathbb{L}_{p, 1}$, donc dans Dom $\delta$, ainsi $\theta$ est-il dans Dom $\delta^{T}$ d'après la proposition 2.11 .

Lorsque $\alpha$ est dans $\mathbb{L}_{p, 1}\left(\tau_{X}^{*} V\right)$, alors le processus $\left(t \rightarrow \alpha(t) \mathbf{1}_{[0, t]}\right)$ appartient à Dom $\delta^{\tau}$. On a alors:

$$
\delta^{\tau}\left(\alpha \mathbf{1}_{[0, t]}\right)=\delta\left(\left(\nabla_{-} X, \mathbf{R} \alpha\right) \mathbf{1}_{[0, t]}\right)+\int_{0}^{t}\left\langle L_{s}^{\tau}, \alpha(s)\right\rangle d s .
$$


On notera $\delta^{\tau}\left(\alpha \mathbf{1}_{[0, t]}\right)=\int_{0}^{t}\left(\left(\nabla_{-} X\right)_{s}, \mathbf{R} \alpha(s)\right) d W_{s}+\int_{0}^{t}\left\langle L_{s}^{\tau}, \alpha(s)\right\rangle d s$.

REMARQUe 4.2. Lorsque le processus $\left(\nabla_{-} X, \mathbf{R} \alpha\right)$ vérifie l'une des conditions suivantes:

i) Il existe $p>1$ tel que $\sup _{t \in[0,1]} E\left(\int_{0}^{1}\left\|D_{s}\left(\nabla_{-} X, \mathbf{R} \alpha\right)_{t}\right\|^{2} d s\right)^{p}<\infty$,

ii) Il existe $p>2$ tel que $E\left(\int_{0}^{1}\left(\int_{0}^{1}\left\|D_{s}\left(\nabla_{-} X, \mathbf{R} \alpha\right)_{t}\right\|^{2} d s\right)^{p} d t\right)<\infty$, le théorème 5.2 de [15] montre que $t \rightarrow \delta^{\tau}\left(\alpha(t) \mathbf{1}_{[0, t]}\right)$ admet une modification continue.

Il est facile de voir que lorsque $X$ satisfait l'hypothèse (Hv) et que $\alpha$ est un champ de 1-formes, alors l'hypothèse [i/] de la Remarque 4.2 est vérifiée. En particulier, la forme d'ordre deux $d \alpha$ est dans le domaine de $\delta^{\tau}$ et de plus le processus $t \mapsto d \alpha \mathbf{1}_{[0, t]}$ est dans $\mathbb{L}_{p, 1}\left(\tau_{X}^{*}(V)\right)$.

\subsection{E.D.S. anticipative}

Dans cette partie nous montrons l'existence et l'unicité de la solution de l'équation différentielle stochastique

$$
d X_{t}=e\left(B_{t}, X_{t}\right) \circ d B_{t} \quad \text { avec } \quad X_{0} \in L^{0}(\Omega, \mathcal{F}, V)
$$

où $e$ est un morphisme de Stratonovitch (Cf. [4]) c'est à dire:

$$
\forall(b, x) \in V \times V ; e(b, x): T_{b} V \rightarrow T_{x} V
$$

est une famille d'applications linéaires qui dépend régulièrement de $(x, y), X_{0}$ est une variable aléatoire à valeurs dans $V$ qui peut anticiper $B$, le processus Brownien sur $V$ conducteur. Nous supposerons dans cette section $d \geq k$ c'est-à-dire que la dimension du Brownien est supérieure à la dimension de la variété, il n'y aura ainsi pas de dégénérescence.

La méthode de D. Ocone et E. Pardoux est maintenant classique: on considère d'abord l'E.D.S. avec une condition initiale déterministe:

$$
d X_{t}(x)=e\left(B_{t}, X_{t}(x)\right) \circ d B_{t} \quad \text { avec } \quad X_{0}(x)=x \in V
$$

qui a une solution unique (Cf. [4])

$$
X_{t}(x)=\int_{0}^{t} e\left(B_{s}, X_{s}(x)\right) \circ d B_{s} .
$$

Puis, nous montrons sous certaines conditions une formule de substitution

$$
\left.\int_{0}^{t} e\left(B_{s}, X_{s}(x)\right) \circ d B_{s}\right|_{x=X_{0}}=\int_{0}^{t} e\left(B_{s}, X_{s}\left(X_{0}\right)\right) \circ d B_{s}
$$


ce qui donnera l'existence de la solution de l'équation (31), où par définition, on dira que $X$ est solution de (31) si pour tout $\eta \in \mathcal{X}^{*}(V)$ et presque tout $t$ :

$$
\int_{0}^{t} \eta\left(X_{s}\right) \circ d X_{s}=\int_{0}^{t} e^{*}(\eta)\left(B_{s}\right) \circ d B_{s} .
$$

A droite de cette égalité on a l'intégrale d'une 1-forme anticipative le long du mouvement Brownien $B$ sur $V$, qui a été définie dans [8] et à gauche, on a l'intégrale de Stratonovitch d'une 1-forme le long d'une $S M A V$, notion qui a été définie dans la section 3 et précisée comme processus dans la remarque 4.2. Nous montrons d'abord un théorème d'existence et d'unicité lorsque $B$ est remplacé par $W$ le processus de Wiener vectoriel:

ThÉoRÈme 4.3. Soit e un morphisme de Stratonovitch, alors l'équation

$$
d X_{t}=e\left(W_{t}, X_{t}\right) \circ d W_{t} \quad \text { avec } \quad X_{0} \in \mathbb{D}_{4,1}(V)
$$

admet une solution unique donnée par $\left.X_{t}(x)\right|_{x=X_{0}}$ où $X_{t}(x)$ est la solution de l'équation

$$
d X_{t}=e\left(W_{t}, X_{t}\right) \circ d W_{t} \quad \text { avec } \quad X_{0}=x \in V .
$$

De plus, $\left.X_{t}(x)\right|_{x=X_{0}}$ est alors une $S M A V$ vérifiant l'hypothèse $(\mathbf{H v})$.

Preuve. Soit $h$ le plongement de Whitney de $V$ dans $\mathbb{R}^{p}$; on note $\widetilde{e}$ le morphisme de Stratonovitch $\widetilde{e}(b, z): T_{b} \mathbb{R}^{d} \rightarrow T_{z} \mathbb{R}^{p}$ défini sur $h(V)$ par

$$
\widetilde{e}(b, h(x))=T_{x} h(e(b, x))
$$

et prolongé sur $\mathbb{R}^{p}$ en étendant les champs de vecteurs $C^{\infty}$ sur un voisinage compact de $h(V)$, puis nuls au-delà (Cf. Elworthy [2] p.286-287).

Lemme 4.4. Sous les hypothèses du Théorème 4.3, l'équation

$$
d Z_{t}=\widetilde{e}\left(W_{s}, Z_{s}\right) \circ d W_{s} \quad \text { avec } \quad Z_{0}=h\left(X_{0}\right)
$$

admet une solution unique qui est $Z_{t}=\phi_{t}\left(X_{0}\right)$ où $\phi$ est le flot solution de l'EDS vectorielle ci-dessous (36).

Preuve. Soit $Z_{t}(z)$ la solution de l'équation

$$
d Z_{s}(z)=\widetilde{e}\left(W_{s}, Z_{s}(z)\right) \circ d W_{s} \quad \text { avec } \quad Z_{0}(z)=z \in h(V) .
$$

Comme le montre Elworthy dans $[2],\left(Z_{t}(z)\right)_{t}$ est un processus à valeurs dans $h(V)$ et l'on peut montrer (Cf. Emery [4]) que pour tout champ de 1-formes $\eta$ sur $\mathbb{R}^{p}$

$$
\int_{0}^{t} \eta\left(Z_{s}(z)\right) \circ d Z_{s}=\int_{0}^{t} \widetilde{e}^{*}\left(W_{s}, Z_{s}(z)\right)(\eta)\left(W_{s}\right) \circ d W_{s} .
$$


Posons $u(s, z)=\widetilde{e}^{*}\left(W_{s}, Z_{s}(z)\right)(\eta)\left(W_{s}\right)$; alors grâce à la régularité de $\widetilde{e}, u(s, z)$ est un processus vectoriel intégrable au sens de Stratonovitch (Cf. [15]). De plus, il est connu (Cf. Kunita [11]) que le flot stochastique solution de (36) est de classe $C^{1}$; comme par construction $\widetilde{e}$ est à support compact dans $\mathbb{R}^{p}$ et que tous les processus en jeu sont continus sur $[0,1] \times \mathbb{R}^{p}$, le processus $u(s, z)$ vérifie les conditions $H_{1}, \ldots, H_{4}$ de la proposition 4.12 de [15].

D'une part la formule de correction Ito-Stratonovitch donne:

$$
\int_{0}^{t} \widetilde{e}^{*}\left(W_{s}, Z_{s}(z)\right)(\eta)\left(W_{s}\right) \circ d W_{s}=\int_{0}^{t} u_{i}(s, z) d W_{s}^{i}+\frac{1}{2} \int_{0}^{t} d\left[u_{i}(\cdot, z), W^{i}\right]_{s}
$$

et suivant la proposition 4.12 de [15]:

$$
\left.\int_{0}^{t} u_{i}(s, z) d W_{s}^{i}\right|_{z=Z_{0}}=\int_{0}^{t} u_{i}\left(s, Z_{0}\right) d W_{s}^{i}+\int_{0}^{t} \partial_{i} u_{l}\left(s, Z_{0}\right) D_{s}^{l} Z_{0}^{i} d s .
$$

Comme $Z_{0} \in \mathbb{D}_{4,1}, D_{s}^{l} Z_{0}^{i}$ peut s'écrire $\frac{1}{2}\left(\nabla_{+}^{l} Z_{0}\right)_{s}$ et ainsi:

$$
\int_{0}^{t} \partial_{i} u_{l}\left(s, Z_{0}\right) D_{s}^{l} Z_{0}^{i} d s+\left.\frac{1}{2} \int_{0}^{t} d\left[u_{i}(\cdot, z), W^{i}\right]_{s}\right|_{z=Z_{0}}=\frac{1}{2} \int_{0}^{t}\left(\nabla_{+}^{i} u_{i}\left(\cdot, Z_{0}\right)\right)_{s} d s
$$

et l'on reconnait la définition de l'intégrale de Stratonovitch d'une 1-forme anticipante le long du mouvement brownien (Cf. [8] ou la proposition 3.2):

$$
\begin{aligned}
\left.\left(\int_{0}^{t} \widetilde{e}^{*}\left(W_{s}, Z_{s}(z)\right)(\eta)\left(W_{s}\right) \circ d W_{s}\right)\right|_{z=Z_{0}} \\
=\int_{0}^{t} \widetilde{e}^{*}\left(W_{s}, Z_{s}\left(Z_{0}\right)\right)(\eta)\left(W_{s}\right) \circ d W_{s}
\end{aligned}
$$

D'autre part, nous devons comparer l'intégrale de Stratonovitch $\int_{0}^{t} \eta\left(Z_{s}(z)\right) \circ$ $d Z_{s}$ évaluée en $z=Z_{0}$ avec l'intégrale de Stratonovitch de la 1 -forme $\eta$ le long de $\left(Z_{t}\left(Z_{0}\right)\right)_{t}$ définie par $Z_{t}\left(Z_{0}\right)=Z_{t}(z)$ évalué en $z=Z_{0}$ une fois vérifié qu'il s'agit bien d'une $S M A R$ vérifiant l'hypothèse $(\mathbf{H})$.

Pour cela il faut identifier les processus $v$ et $z$ tels que:

$$
Z_{t}\left(Z_{0}\right)=Z_{0}+\int_{0}^{t} v_{s} d W_{s}+\int_{0}^{t} z_{s} d s
$$

L'équation (36) et la proposition 4.12 de [15] appliquée au processus matriciel $\widetilde{e}\left(W_{s}, Z_{s}(z)\right)$ montrent que

$$
v_{s}=\widetilde{e}\left(W_{s}, Z_{s}\left(Z_{0}\right)\right) ; z_{s}=\partial_{i}\left(\widetilde{e} \circ Z_{s}\right)_{j} D_{s}^{j} Z_{0}^{i}+\left.\frac{1}{2} \frac{d}{d s}\left[\widetilde{e}\left(W_{s}, Z_{s}(z)\right), W_{s}\right]\right|_{z=Z_{0}} .
$$


La régularité de $\widetilde{e}$ et la compacité de son support montrent que $Z$ est bien une $S M A R$ vérifant l'hypothèse $(\mathbf{H})$.

Utilisant la proposition 3.2 , on obtient:

$$
\begin{aligned}
\int_{0}^{t} \eta \circ d Z_{s}\left(Z_{0}\right)= & \int_{0}^{t} v_{s}^{*}\left(\eta\left(Z_{s}\left(Z_{0}\right)\right)\right) d W_{s}+\int_{0}^{t}\left(z_{s}, \eta\left(Z_{s}\left(Z_{0}\right)\right)\right) d s \\
& +\frac{1}{2} \int_{0}^{t}\left(\nabla_{+}^{j} \eta_{l}\left(Z_{.}\left(Z_{0}\right)\right)\right)_{s} \cdot\left(\nabla_{-}^{j} Z_{.}^{l}\left(Z_{0}\right)\right)_{s} d s .
\end{aligned}
$$

Les deux premiers termes des membres de droite de (38) et (41) sont identiques.

Par ailleurs, l'intégrand du terme à variations finies à droite de l'équation (41) se détaille de la façon suivante

$$
\begin{aligned}
& \left(z_{s}, \eta\left(Z_{s}\left(Z_{0}\right)\right)\right)+\frac{1}{2}\left(\nabla_{+}^{j} \eta_{l}\left(Z_{.}\left(Z_{0}\right)\right)\right)_{s} \cdot\left(\nabla_{-}^{j} Z_{.}^{l}\left(Z_{0}\right)\right)_{s} \\
& =\eta_{l}\left(Z_{s}\left(Z_{0}\right)\right) \partial_{i}\left(\widetilde{e}^{l} \circ Z_{s}\right)_{j} D_{s}^{j} Z_{0}^{i}+\frac{1}{2} \eta_{l}\left(Z_{s}\left(Z_{0}\right)\right) \frac{d}{d s}\left[\widetilde{e}\left(W_{s}, Z_{s}(z)\right), W_{s}\right]_{s}^{l} \\
& \quad+\frac{1}{2}\left(\nabla_{+}^{j} \eta_{l}\left(Z \cdot\left(Z_{0}\right)\right)\right)_{s} \cdot\left(\nabla_{-}^{j} Z_{.}^{l}\left(Z_{0}\right)\right)_{s} .
\end{aligned}
$$

Or, puisque de manière générale, $\left(\nabla_{+}^{j} u\right)_{s}=\left(\nabla_{-}^{j} u\right)_{s}+2\left(D_{-}^{j} u\right)_{s}$, il vient:

$$
\left.\nabla_{+}^{j} \eta_{l}\left(Z_{.}\left(Z_{0}\right)\right)\right)_{s}=\partial_{i} \eta_{l}\left(\nabla_{-}^{j}\left(Z_{.}^{i}\left(Z_{0}\right)\right)\right)_{s}+2 \partial_{i}\left(\eta_{l} \circ Z_{s}\right)\left(D_{-} Z_{0}^{i}\right)_{s}^{j} .
$$

En remarquant que $\left(\nabla_{-}^{j} Z_{.}^{l}\left(Z_{0}\right)\right)_{s}=v_{s, j}^{l}$ et en regroupant le premier terme à droite de $(42)$ avec la deuxième partie de $(43)$ on reconnait:

$$
\begin{aligned}
& \eta_{l}\left(Z_{s}\left(Z_{0}\right)\right) \partial_{i}\left(\widetilde{e} \circ Z_{s}\right)_{j}^{l} D_{s}^{j} Z_{0}^{i}+\partial_{i}\left(\eta_{l} \circ Z_{s}\right)\left(\widetilde{e} \circ Z_{s}\right)_{j}^{l} D_{s}^{j} Z_{0}^{i} \\
& \quad=\partial_{i}\left(\eta \circ Z_{s}\left(Z_{0}\right),\left(\widetilde{e} \circ Z_{s}\right)_{j}\left(Z_{0}\right)\right) D_{s}^{j} Z_{0}^{i} .
\end{aligned}
$$

On identifie alors le reste de (42):

$$
\begin{gathered}
\left.\frac{1}{2} \eta_{l}\left(Z_{s}\left(Z_{0}\right)\right) \frac{d}{d s}[\widetilde{e}(W, Z(z)), W]_{s}^{l}\right|_{z=Z_{0}}+\frac{1}{2} \partial_{i} \eta_{l}\left(Z_{s}\left(Z_{0}\right)\right) \tilde{e}_{j}^{i}(W, Z(z)) \widetilde{e}_{j}^{l}(W, Z(z)) \\
=\frac{1}{2} \frac{d}{d s}\left[\eta_{l}\left(Z .\left(Z_{0}\right)\right) \widetilde{e}_{j}^{l}, W^{j}\right]_{s}
\end{gathered}
$$

Regroupant ces deux dernières égalités, l'intégrale (41) se récrit:

$$
\begin{aligned}
\int_{0}^{t} \eta \circ d Z_{s}\left(Z_{0}\right)= & \int_{0}^{t} \eta_{l}\left(Z_{s}\left(Z_{0}\right)\right) \widetilde{e}_{j}^{l}(W, Z(z)) d W_{s}^{j} \\
& +\int_{0}^{t} \partial_{i}\left(\eta \circ Z_{s}\left(Z_{0}\right),\left(\widetilde{e} \circ Z_{s}\right)_{j}\left(Z_{0}\right)\right) D_{s}^{j} Z_{0}^{i} d s \\
& +\frac{1}{2} d\left[\eta_{l}\left(Z .\left(Z_{0}\right)\right) \widetilde{e}_{j}^{l}, W^{j}\right]_{s},
\end{aligned}
$$


soit exactement l'expression trouvée en (40).

Ainsi, nous avons donc montré que pour tout $\eta$ et pour tout $t$ :

$$
\int_{0}^{t} \eta\left(Z_{s}\left(Z_{0}\right)\right) \circ d Z_{s}=\int_{0}^{t} \widetilde{e}^{*}\left(W_{s}, Z_{s}\left(Z_{0}\right)\right)(\eta)\left(W_{s}\right) \circ d W_{s}
$$

c'est-à-dire que le processus $Z_{t}\left(Z_{0}\right)$ est solution de l'E.D.S. anticipante (35).

Remarquons que l'on a montré de plus au cours de cette démonstration la formule de substitution annoncée

$$
\int_{0}^{t} \eta\left(Z_{s}\left(Z_{0}\right)\right) \circ d Z_{s}=\left.\int_{0}^{t} \eta\left(Z_{s}(z)\right) \circ d Z_{s}\right|_{z=Z_{0}} .
$$

La solution $\left(Z_{t}(z)\right)_{t}$ est un flot de difféomorphismes (Cf. Kunita [11]) sur $h(V)$, c'està-dire que $Z_{t}(\omega, z)=\varphi_{t}(\omega)(z)$ où $\varphi_{t}: h(V) \rightarrow h(V)$ p.s. Ainsi $Z_{t}\left(Z_{0}\right)=\varphi_{t}\left(Z_{0}\right)$ est un processus qui vit dans $h(V)$ presque sûrement et il existe un processus $Y_{t}$ sur $V$ tel que $h\left(Y_{t}\right)=Z_{t}$ p.s. D'autre part, l'équation (34) admet une unique solution $X_{t}(x)$ pour tout $x$ de $V$. Donc $h\left(X_{t}(x)\right)=Z_{t}(z)$ (avec $z=h(x)$ ) solution de l'équation (36). Ainsi, pour $Z_{0}=h\left(X_{0}\right), Z_{t}\left(Z_{0}\right)=\left.h\left(X_{t}(x)\right)\right|_{x=X_{0}}$. L'injectivité de $h$ montre alors que $Y_{t}=X_{t}\left(X_{0}\right)$ et par conséquent l'unicité de la solution $Y$.

On déduit immédiatement par composition d'applications que $X_{t}\left(X_{0}\right)$ est une $S M A V$ vérifiant l'hypothèse $(\mathbf{H v})$ de ce que $Z$ est une $S M A R$ vérifiant l'hypothèse $(\mathbf{H})$. Il reste à montrer, pour la preuve complète du théorème, que le processus $\left(X_{t}\left(X_{0}\right)\right)_{t}$ est solution de l'équation (33). On doit donc montrer que pour tout champ de 1 -formes $\theta$ sur $V$, on a:

$$
\int_{0}^{t} \theta\left(X_{s}\left(X_{0}\right)\right) \circ d X_{s}=\int_{0}^{t} e^{*}\left(W_{s}, X_{s}\left(X_{0}\right)\right)(\theta)\left(W_{s}\right) \circ d W_{s}
$$

l'intégrale de gauche est l'intégrale de Stratonovitch de la 1-forme le long du processus anticipant $X_{t}\left(X_{0}\right)$; l'intégrale de droite est l'intégrale de Stratonovitch de la 1-forme anticipante $\widetilde{e}^{*}\left(W_{s}, X_{s}\left(X_{0}\right)\right) \theta\left(W_{s}\right)$ le long de $W$ (Cf. [8]).

Le membre de gauche de $(48)$ s'écrit $S^{\tau, X\left(X_{0}\right)}(\theta)$ et l'on a montré dans le Corollaire 3.4 que:

$$
S^{\tau, X\left(X_{0}\right)}(\theta)=S^{\tau, Z\left(h\left(X_{0}\right)\right)}(\widetilde{\theta})
$$

où $\widetilde{\theta}=T h^{*}(\theta)$. L'égalité (47) permet d'écrire

$$
S^{\tau, Z\left(h\left(X_{0}\right)\right)}(\widetilde{\theta})=\left.S^{\tau, Z(z)}(\widetilde{\theta})\right|_{z=h\left(X_{0}\right)}
$$

mais $S^{\tau, Z(z)}(\widetilde{\theta})=S^{\tau, X(x)}(\theta)$ avec $h(x)=z$. Par l'injectivité de $h$, si $z=h\left(X_{0}\right)$ alors $x=X_{0}$ d'où $\left.S^{\tau, Z(z)}(\widetilde{\theta})\right|_{z=h\left(X_{0}\right)}=\left.S^{\tau, X\left(X_{0}\right)}(\theta)\right|_{x=X_{0}}$, ce qui permet d'écrire

$$
S^{\tau, X\left(X_{0}\right)}(\theta)=\left.S^{\tau, X(x)}(\theta)\right|_{x=X_{0}} .
$$


D'autre part, en notant $u(s, x)=e^{*}\left(W_{s}, X_{s}(x)\right)(\theta)\left(W_{s}\right)$, le terme de droite de (48) est égal à $S^{\tau, W}\left(u\left(\cdot, X_{0}\right)\right)$, c'est à dire:

$$
S^{\tau, W}\left(u\left(\cdot, X_{0}\right)\right)=\delta\left(u\left(\cdot, X_{0}\right)\right)+\frac{1}{2} \int_{0}^{t}\left(\nabla_{+} u\left(\cdot, X_{0}\right)\right)_{s} d s
$$

et, une modification évidente de la proposition 4.11 de [15] permet de montrer une formule de substitution dans le cas où le paramètre prend ses valeurs dans une variété compacte; d'où

$$
\left.\int_{0}^{t} u_{j}(s, x) d W_{s}^{j}\right|_{x=X_{0}}=\int_{0}^{t} u_{j}\left(s, X_{0}\right) d W_{s}^{j}+\frac{1}{2} \int_{0}^{t} \partial_{l} u_{j}\left(s, X_{0}\right) D_{s}^{j} X_{0}^{l} d s .
$$

De plus, comme en (39):

$$
\left.\int_{0}^{t}\left(\nabla_{+}^{j} u_{j}\left(\cdot, X_{0}\right)\right)_{s} d s=\int_{0}^{t} \partial_{l} u_{j}\left(s, X_{0}\right) \cdot\left(\nabla_{+}^{j} X_{0}^{l}\right)_{s} d s+\int_{0}^{t} \partial_{j} u_{j}\left(s, X_{0}\right)\right) d s
$$

d'où

$$
\begin{aligned}
S^{\tau, W}\left(u\left(\cdot, X_{0}\right)\right) & \left.=\left.\int_{0}^{t} u_{j}(s, x) d W_{s}^{j}\right|_{x=X_{0}}+\frac{1}{2} \int_{0}^{t} \partial_{j} u_{j}\left(s, X_{0}\right)\right) d s \\
& =\left.\int_{0}^{t} u_{j}(s, x) \circ d W_{s}^{j}\right|_{x=X_{0}} \\
& =\left.\int_{0}^{t} e^{*}\left(W_{s}, X_{s}(x)\right)(\theta)\left(W_{s}\right) \circ d W_{s}\right|_{x=X_{0}} .
\end{aligned}
$$

Pour obtenir l'égalité (48), il suffit de combiner (49) et (50) et de remarquer que pour tout $x$ de $V$

$$
\int_{0}^{t} \theta\left(X_{s}(x)\right) \circ d X_{s}=\int_{0}^{t} e^{*}\left(W_{s}, X_{s}(x)\right)(\theta)\left(W_{s}\right) \circ d W_{s}
$$

puisque $\left(X_{t}(x)\right)_{t}$ est solution de l'E.D.S. (34).

Corollaire 4.5. Sous les hypothèses du Théorème 4.3, soit $B_{t}$ le mouvement Brownien sur $V$ et $X_{t}(x)$ la solution de l'E.D.S.

$$
d X_{t}(x)=f\left(B_{t}, X_{t}(x)\right) \circ d B_{t} \quad \text { avec } \quad X_{0}=x \in V .
$$

Alors $X_{t}\left(X_{0}\right)$ est solution de l'E.D.S. anticipante

$$
d X_{t}\left(X_{0}\right)=f\left(B_{t}, X_{t}\left(X_{0}\right)\right) \circ d B_{t} \quad \text { avec } \quad X_{0} \in \mathbb{D}_{4,1}(V) .
$$


Preuve. L'équation (52) se traduit par:

$$
\text { pour tout } \eta \in \mathcal{X}^{*}(V), \int_{0}^{t} \eta \circ d X_{s}=\int_{0}^{t} f^{*}\left(B_{s}, X_{s}(x)\right)(\eta) \circ d B_{s},
$$

c'est à dire que $S^{B}\left(f^{*}(\eta)\right)=S^{X}(\eta)$.

On se ramène comme précédemment par le plongement de Whitney $h$ au mouvement Brownien vectoriel $W=h(B)$ en utilisant le corollaire 3.4 qui montre que $S^{B}\left(f^{*}(\eta)\right)=S^{W}\left(\widetilde{f^{*}(\eta)}\right)$ où $(T h)^{*}\left(\widetilde{f^{*}(\eta)}\right)=f^{*}(\eta)$. Il suffit alors d'identifier un morphisme de Stratonovitch $e$ qui vérifie $e^{*}(\eta)=\widetilde{f^{*}(\eta)}$. Un tel morphisme est défini sur $h(V) \times V$ par:

$$
\begin{aligned}
e(h(b), x): T_{h(b)} \mathbb{R}^{d} & \rightarrow T_{x} V \\
T h(A) & \mapsto f(b, x) A
\end{aligned}
$$

et prolongé sur tout $\mathbb{R}^{d} \times V$ de façon régulière.

\subsection{Approximations}

Nous montrons dans cette partie un théorème d'approximation de la solution de l'E.D.S. anticipante dont l'existence a été prouvée dans le corollaire 4.5. Il s'agit donc de l'équation

$$
d X_{t}\left(X_{0}\right)=f\left(B_{t}, X_{t}\left(X_{0}\right)\right) \circ d B_{t} \quad \text { avec } \quad X_{0} \in \mathbb{D}_{4,1}(V)
$$

où $B$ est le mouvement Brownien sur $V$ (Cf. [9], [8]) et la condition initiale $X_{0}$ peut anticiper le processus conducteur. Les champs $f$ sont $C^{\infty}$.

La méthode d'approximation est analogue à celle utilisée dans la section 3.2. Soit $I(x, y, t)$ une règle d'interpolation sur $V$ (Cf. [4]) telle que pour tout $(x, y) \in$ $V \times V, I(x, y, \cdot)$ soit une courbe régulière de $x$ à $y$ vérifiant, uniformément sur tout compact en temps

$$
\frac{\partial^{m}}{\partial t^{m}} I(x, y, t)=O\left[d^{m}(x, y)\right], \quad m=1,2,3 .
$$

Soit $\sigma=\left\{0=T_{1}, \ldots, T_{n}=1\right\}$ une subdivision de $[0,1]$ dont le pas $\sup _{l}\left|T_{l+1}-T_{l}\right|$ tend vers zéro lorsque $n \rightarrow \infty$. On note $\Delta_{l} T=\left|T_{l+1}-T_{l}\right|$ et $B_{t}^{n}$ le processus interpolant $B$ :

$$
B_{t}^{n}=I\left(B_{T_{l}}, B_{T_{l+1}}, \frac{t-T_{l}}{\Delta_{l} T}\right) \text { pour } t \in\left[T_{l} ; T_{l+1}\right] \text { et } l=1, \ldots, n .
$$

DÉfinition 4.6. On note $X_{t}^{n}$ le processus approchant $X$; c'est la solution de l'équation différentielle ordinaire

$$
d X_{t}^{n}(x)=f\left(B_{t}^{n}, X_{t}^{n}(x)\right) \circ d B_{t}^{n} \quad \text { avec } \quad X_{0}^{n}=x \in V .
$$


THÉORÈme 4.7. Soit $X$ solution de (55); alors $X_{t}^{n}\left(X_{0}\right)$ converge lorsque $n$ tend vers l'infini vers $X_{t}\left(X_{0}\right)$ dans $L^{r}$ uniformément en temps.

Preuve. Utilisant le lemme (7.26) de [4], et le plongement de Whitney $h$ de $V$ dans $\mathbb{R}^{p}$, on a l'existence d'une interpolation $J$ sur $\mathbb{R}^{p}$ telle que:

$$
J(h(x), h(y), t)=h \circ I(x, y, t) .
$$

On note $W^{n}=h\left(B^{n}\right)$, on a donc

$$
W_{t}^{n}=J\left(W_{T_{l}}, W_{T_{l+1}}, \frac{t-T_{I}}{\Delta_{l} T}\right) \text { pour } t \in\left[T_{l} ; T_{l+1}\right] \text { et } l=1, \ldots, n
$$

avec $W=h(B) . W$ est un processus de Wiener sur $h(V)$ que l'on prolonge par un processus de Wiener dans $\mathbb{R}^{p}$. Les champs de vecteurs $f$ étant tangents à $V$, les champs $\widetilde{f}=T h(f)$ sont tangents à $h(V)$ donc la solution $Z_{t}^{n}(z)$ de l'équation

$$
d Z_{t}^{n}(z)=\tilde{f}\left(W_{t}^{n}, Z_{t}^{n}(z)\right) \circ d W_{t}^{n} \quad \text { avec } \quad Z_{0}^{n}=z \in h(V)
$$

reste p.s. dans $h(V)$. On sait (Cf. Kunita [11] Théorème 5.7.3 et exemple suivant) que si le processus $B^{n}$ (resp. $W^{n}$ restreint à $h(V)$ ) converge fortement vers $B$ (resp. $W$ restreint à $h(V))$ alors la solution $Z^{n}$ converge fortement

$$
d Z_{t}(z)=\tilde{f}\left(W_{t}, Z_{t}(z)\right) \circ d W_{t} \quad \text { avec } \quad Z_{0}=z \in h(V)
$$

c'est-à-dire que

$$
E\left(\sup _{(t, z) \in[0 ; 1] \times h(V)}\left|Z_{t}^{n}(z)-Z_{t}(z)\right|^{r}\right) \stackrel{n \rightarrow \infty}{\longrightarrow} 0 .
$$

Soit alors $Z_{0}$ une variable aléatoire prenant ses valeurs dans $h(V)$. On note $Z_{t}^{n}\left(Z_{0}\right)$ la solution de l'équation

$$
d Z_{t}^{n}(z)=\tilde{f}\left(W_{t}^{n}, Z_{t}^{n}(z)\right) \circ d W_{t}^{n} \quad \text { avec } \quad Z_{0}^{n}=Z_{0} \in h(V)
$$

et $Z_{t}\left(Z_{0}\right)$ celle de l'équation

$$
d Z_{t}(z)=\tilde{f}\left(W_{t}, Z_{t}(z)\right) \circ d W_{t} \quad \text { avec } \quad Z_{0} \in L^{\circ}(\Omega, \mathcal{F}, h(V)) .
$$

On a alors p.s.

$$
\left|Z_{t}^{n}\left(Z_{0}\right)-Z_{t}\left(Z_{0}\right)\right|^{r} \leq \sup _{z \in h(V)}\left|Z_{t}^{n}(z)-Z_{t}(z)\right|^{r}
$$

donc

$$
E\left(\sup _{t \in[0 ; 1]}\left|Z_{t}^{n}\left(Z_{0}\right)-Z_{t}\left(Z_{0}\right)\right|^{r}\right) \leq E\left(\sup _{(t, z) \in[0 ; 1] \times h(V)}\left|Z_{t}^{n}(z)-Z_{t}(z)\right|^{r}\right)
$$


Donc $Z_{t}^{n}\left(Z_{0}\right) \stackrel{n \rightarrow \infty}{\longrightarrow} Z_{t}\left(Z_{0}\right)$ dans $L^{r}$, uniformément en temps. D'où l'on déduit, $h$ étant injective, que $X_{t}^{n}\left(X_{0}\right)$ converge vers $X_{t}\left(X_{0}\right)$ dans $L^{r}$, uniformément en temps.

\section{References}

[1] R. Buckdahn, Linear skorohod stochastic differential equations, Proba. Th. Rel. Fields, 90 (1991), 223-240.

[ 2 ] K.D. Elworthy, Differential Equations on Manifolds, in Ecoles d'Eté de Probabilité de StFlour XV-XVII, 1985-1987; Ed. P.L. Hennequin, Lecture Notes In Mathematics 1362, Springer-Verlag, 1989.

[ 3 ] K.D. Elworthy, Stochastic Differential Equations on Manifolds, London Math. Soc. Lect. Note Ser., 70, Cambridge University Press (1982).

[4] M. Emery, Stochastic Calculus on Manifolds, Universitext, Springer-Verlag Publ. Berlin, 1989.

[ 5 ] S. Fang and P. Malliavin, Stochastic Analysis on the Path Manifolds, J. Functional Analysis, 249-274, 1993.

[6] A. Grorud, Un crochet non symétrique en calcul stochastique anticipatif, Proceedings of the $2^{\text {nd }}$ Workshop on Stochastic Analysis, Silivri, 1988, L.N. 1444, 183-192, Springer.

[ 7 ] A. Grorud and M. Pontier, Un Calcul Anticipatif sur une variété riemannienne compacte, Stochastic Analysis and related topics, H. Korezlioglu and S. Ustunel ed., Progress in Probability 31, Birkhausen, Boston, 1992.

[ 8 ] A. Grorud and M. Pontier, Calcul anticipatif d'ordre deux, Stochastics and Stochastic Reports, 42 (1993), 1-23.

[ 9 ] N. Ikeda and S. Watanabe, Stochastic Differential Equations and diffusion processes, North Holland, Amsterdam, 1981.

[10] M. Jolis and M. Sanz, Integrator properties of the Skorohod Integral, Preprint.

[11] H. Kunita, Stochastic Flows and Stochastic Differential Equations, Cambridge University Press, Cambridge, 1990.

[12] P.A. Meyer, Géométrie stochastique sans larmes, Séminaire de Probabilités XV, Lecture Notes in Math., 850, Springer, 1981.

[13] Norris, Stochastic Calculus on Manifolds, Preprint.

[14] D. Nualart, Stochastic Calculus for Anticipating Processes, Publications del Departament d'Estadistica, Universitat de Barcelona, 1990.

[15] D. Nualart and E. Pardoux, Stochastic calculus with anticipating integrands, Proba. Th. Rel. Fields, 78 (1988), 535-581.

[16] D. Nualart and M. Zakai, Generalized Stochastic Integrals and the Malliavin calculus, Proba. Th. Rel. Fields, 73 (1986), 255-280.

[17] D. Ocone and E. Pardoux, A generalized Ito-Ventzell formula, Application to a class of anticipating stochastic differential equations, Ann. Inst. H. Poincaré, 25-1 (1989), 39 71.

[18] D. Ocone and E. Pardoux, Linear stochastic differential equations with boundary conditions, Proba. Th. Rel. Fields, 82 (1989), 489-526.

[19] F. Russo and P. Vallois, Non causal Stochastic Integration for LAD LAG processes, preprint, 1993.

[20] L. Schwartz, Géométrie Différentielle du second ordre, Semi-martingales et Equations Différentielles sur une variété différentiable, in Séminaire de Probabilités XVI, Lectures Notes in Math., 921, Springer-Verlag, 1982.

[21] J. Weidman, Linear Operators in Hilbert Spaces, Springer-Verlag, 68, Berlin, 1980. 
Axel Grorud

U.R.A. CNRS 225

Université de Provence, CMI

39, Rue Toliot Curie

13453 Marseille, France

Monique Pontier

Dp. Mathématiques

UNIVERSITÉ D'ORLÉANS

B.P. 6759

45067 Orleans, France 